\title{
Fibres non réduites d'un schéma arithmétique
}

\author{
Chunhui Liu ${ }^{1}$
}

Published online: 9 March 2021

(C) The Author(s) 2021

\section{Résumé}

Pour un schéma réduit projectif sur l'anneau des entiers d'un corps de nombres, l'ensemble des places au dessus desquelles les fibres du schéma ne sont pas réduite est un ensemble fini. On donne une majoration explicite du produit des normes de ces places. Pour cela, on introduit une généralisation de la notion de hauteur sur l'anneau adélique. En utilisant la théorie des variétés de Chow, on ramène le cas général d'un schéma de dimension pure à celui d'une hypersurface et on traite ce dernier à l'aide du résultant de l'équation de l'hypersurface et des dérivées partielles de cette équation.

\begin{abstract}
(Non-reduced fibers of an arithmetic scheme) For a reduced projective scheme over the ring of integers of a number field, the set of places over which the fibres of the scheme are not reduced is a finite set. We give an explicit upper bound for the product of the norms of places in this set. For this purpose, we introduce a generalization of the notion of height over the adelic ring. We reduce the general case of a scheme of pure dimension to the case of a hypersurface by using the theory of Chow varieties. The case of a hypersurface is then treated with the help of the resultant of the equation of the hypersurface with some partial derivatives of the equation.
\end{abstract}

\section{Contents}

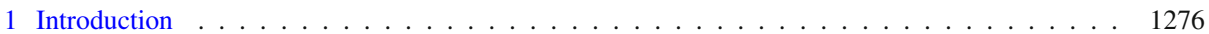

1.1 Résultat principal . . . . . . . . . . . . . . . . . . . . . . . . . . 1276

1.2 Méthode . . . . . . . . . . . . . . . . . . . . . . . . . . . 1277

1.3 Organisation de l'article . . . . . . . . . . . . . . . . . . . . . . . . . . . . 1277

Remerciements . . . . . . . . . . . . . . . . . . . . . . . . . . . 1277

2 La variété de Chow et la variété de Cayley . . . . . . . . . . . . . . . . . . . . . . . . . . . 1277

2.1 La formation sur un corps . . . . . . . . . . . . . . . . . . . . . . . . . . 1278

2.2 Le diviseur de Cayley sur un anneau de Dedekind . . . . . . . . . . . . . . . . . . . . . . . 1280

3 Hauteurs d'un schéma projectif . . . . . . . . . . . . . . . . . . . . . . . . . . 1283

3.1 Préliminaires . . . . . . . . . . . . . . . . . . . . . . . . . . . . . . . . . 1284

Fibré vectoriel normé . . . . . . . . . . . . . . . . . . . . . . . . . . . . . . 1284

Fonction hauteur . . . . . . . . . . . . . . . . . . . . . . . . . 1284

Chunhui Liu

chunhui.liu@hit.edu.cn

1 Institute for Advanced Study in Mathematics, Harbin Institute of Technology, Harbin 150001, People's Republic of China 
3.2 Hauteurs d'un schéma projectif de dimension pure . . . . . . . . . . . . . . . . . . . . . . . . 1284

3.3 Hauteur d'une hypersurface projective . . . . . . . . . . . . . . . . . . . . . . . . . . 1285

Hauteur de la variété de Cayley . . . . . . . . . . . . . . . . . . . . . . . . . . . . . . . . . 1287

Sur la comparaison des hauteurs . . . . . . . . . . . . . . . . . . . . . . . . . . . . 1287

3.4 Hauteur adélique . . . . . . . . . . . . . . . . . . . . . . . . . . . . . . . . . . . . . 1289

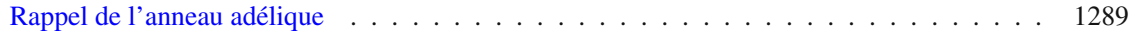

Hauteur sur l'anneau adélique . . . . . . . . . . . . . . . . . . . . . . . . . . . . . . . 1289

4 Une estimation de l'annulation de résultant par réductions . . . . . . . . . . . . . . . . . . 1291

4.1 Résultant sur un anneau . . . . . . . . . . . . . . . . . . . . . . . . . . . . . . . . . . . . . 1291

4.2 Polynôme adéliquement primitif . . . . . . . . . . . . . . . . . . . . . . . . . . . . 1292

4.3 L'estimation d'une hauteur du résultant . . . . . . . . . . . . . . . . . . . . . . . . . . . . . 1293

5 Contrôle des fibres non réduites d'une hypersurface projective . . . . . . . . . . . . . . . . . . 1296

5.1 Résultats préliminaires . . . . . . . . . . . . . . . . . . . . . . . . . . . 1296

Critère du réduisant d'une hypersurface . . . . . . . . . . . . . . . . . . . . . . . . . . . 1296

Changement de coordonnée . . . . . . . . . . . . . . . . . . . . . . . . . . . . . . . 1297

5.2 Description numérique des fibres non réduites . . . . . . . . . . . . . . . . . . . . . . . 1298

6 Contrôle des fibres non réduites d'un schéma de dimension pure . . . . . . . . . . . . . . . . . 1300

References . . . . . . . . . . . . . . . . . . . . . . . . 1302

\section{Introduction}

Soit $X \rightarrow \operatorname{Spec} \mathcal{O}_{K}$ un schéma noethérien réduit, où $K$ est un corps de nombres et $\mathcal{O}_{K}$ est l'anneau des entiers de $K$. On désigne par $\operatorname{Spm} \mathcal{O}_{K}$ l'ensemble des idéaux maximaux de l'anneau $\mathcal{O}_{K}$. Une place $\mathfrak{p} \in \operatorname{Spm} \mathcal{O}_{K}$ est appelée place non réduite du schéma $X \rightarrow \operatorname{Spec} \mathcal{O}_{K}$ si la fibre $X_{\mathbb{F}_{\mathfrak{p}}}=X \times{ }_{\operatorname{Spec}} \mathcal{O}_{K} \operatorname{Spec} \mathbb{F}_{\mathfrak{p}} \rightarrow \operatorname{Spec} \mathbb{F}_{\mathfrak{p}}$ n'est pas réduite, où $\mathbb{F}_{\mathfrak{p}}$ est le corps résiduel de $\mathcal{O}_{K}$ par rapport à p. D'après [13, Théorème (9.7.7)], il n'y a qu'un nombre fini d'idéaux maximaux $\mathfrak{p} \in \operatorname{Spm} \mathcal{O}_{K}$ tels que la fibre $X_{\mathbb{F}_{\mathfrak{p}}} \rightarrow \operatorname{Spec} \mathbb{F}_{\mathfrak{p}}$ ne soit pas réduite.

Il est naturel de considérer une description numérique des places non réduites. Par exemple, on considère la majoration du nombre de ces idéaux maximaux ou la majoration du produit des normes de ces idéaux maximaux.

Erné a considéré un sujet similaire. Dans [6], étant donnée une hypersurface projective géométriquement intègre d'un degré fixé, par le théorème arithmétique de Bézout introduit dans [1, Theorem 5.4.4, Theorem 5.5.1], elle étudie la majoration du produit des normes de idéaux maximaux tels que les fibres contiennent une hypersurface d'un autre degré plus petit fixé. Dans [7], elle étudie le cas de schéma projectif géométriquement intègre en utilisant la théorie des variétés de Chow.

\subsection{Résultat principal}

Dans cet article, pour un schéma projectif réduit sur un corps de nombres arbitraire, on donnera une majoration du produit des normes des idéaux maximaux non réduits.

Theorem 1.1 (Théorème 6.1) Soit $X$ un sous-schéma fermé réduit de dimension pure d et de degré $\delta$ de $\mathbb{P}_{K}^{n}$, dont l'adhérence schématique dans $\mathbb{P}_{\mathcal{O}_{K}}^{n}$ est $\mathscr{X}$. On désigne par $\mathcal{Q}(\mathscr{X})$ l'ensemble des places sur lesquelles les fibres de $\mathscr{X}$ ne sont pas réduites. Alors on a

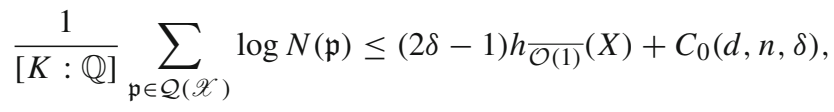

où $N(\mathfrak{p})=\#\left(\mathcal{O}_{K} / \mathfrak{p}\right)$, et $h_{\overline{\mathcal{O}(1)}}(X)$ est une hauteur de $X$ (voir la Définition 3.1). De plus, on explicitera la constant $C_{0}(d, n, \delta)$ dans le Théorème 6.1. 


\subsection{Méthode}

D'abord on résout ce problème dans le cas où $X$ est une hypersurface projective (voir le Théorème 5.5). On considère le polynôme homogène qui définit $X$ comme un polynôme à coefficients dans l'anneau adélique $\mathbb{A}_{K}$ (voir la Remarque 3.12). Dans ce cas-là, on considère son polynôme primitif au sens adélique. De plus, on considère un résultant de ce polynôme primitif, qui est non-nul lorsque l'hypersurface $X$ est réduite. On donnera une majoration des idéaux maximaux tels que les réductions du polynôme modulo lequels s'annulent, ce qui donne un contrôle des places non réduites.

En suite, on résout le cas où $X$ est un schéma projectif de dimension pure en utilisant la théorie des variétés de Chow et des variétés de Cayley (voir le Théorème 6.1). Si un schéma de dimension pure est réduit, toute composante irréductible de sa forme de Chow ou de sa variété de Cayley est de multiplicité 1 (voir la Définition 2.1).

La méthode dans cet article est différente de celle dans [6,7] et permet d'obtenir des résultats explicits. Comparée aux estimations dans [6,7], notre majoration donne une meilleure dépendance de la hauteur de $X$ et, globalement, de meilleures constantes. Comme on utilise une méthode explicite, il faut utiliser la hauteur classique (voir la Définition 3.2) directement. Pour le cas général de dimension pure, on a besoin de comparer certaines hauteurs de $X$.

\subsection{Organisation de l'article}

Cet article est organisé comme suivant. Dans la section 2, on rappellera la théorie des variétés de Chow et des variétés de Cayley, qui est purement géométrique. Dans la section 3, on comparera quelques hauteurs d'un schéma arithmétique de dimension pure. Dans la section 4 , on construira un résultant particulier, et donnera une majoration des idéaux maximaux de $\mathcal{O}_{K}$ tels que les réductions de ce résultant modulo lesquels sur laquelles s'annulent. Dans la section 5, on donnera la majoration mentionnée plus haut pour le cas d'une hypersurface dans le Théorème 5.5, où l'on considère le résultant de l'équation qui définit l'hypersurface. Dans la section 6, on traitera le cas d'un schéma de dimension pure général par la théorie des variétés de Chow et des variétés de Cayley au Théorème 6.1.

\section{Remerciements}

Ce travail fait partie de la thèse de l'auteur préparée à l'Université Paris Diderot - Paris 7. L'auteur voudrait remercier profondément ses directeurs de thèse Huayi Chen et Marc Hindry pour leurs suggèstions autour de ce travail. De plus, l'auteur voudrait remercier le rapporteur anonyme pour sa lecture attentive et ses nombreuses suggestions qui ont permis d'améliorer grandement le présent texte.

\section{La variété de Chow et la variété de Cayley}

Dans cette section, on rappellera les notions de variété de Chow et de variété de Cayley d'un schéma projectif de dimension pure. Dans tout l'article, les anneaux considérés sont commutatifs et unitaires sauf mention contraire. 


\subsection{La formation sur un corps}

Pour la construction des variétés de Chow et des variétés de Cayley sur un corps, on utilise une approche inspirée par [4, \$3.1]. On renvoie à [11] pour une introduction systématique de cette théorie.

f Soient $A$ un anneau, et $M$ un $A$-module. On désigne par $\ell_{A}(M)$ la longueur de $M$ comme un $A$-module. On revoie les lecteurs à [5, §2.4] pour plus de détails.

Soit $m$ un entier positif. On désigne par $\operatorname{Sym}_{A}^{m}(M)$ le $m$-ième produit symétrique de $M$, ou par $\operatorname{Sym}^{m}(M)$ s'il n'y a pas d'ambiguïté sur $A$. De plus, on désigne par

$$
\operatorname{Sym}_{A}(M):=\bigoplus_{i \in \mathbb{N}} \operatorname{Sym}_{A}^{i}(M) .
$$

La notion introduite au-dessous provient de $[10, \S 1.5]$.

Definition 2.1 Soient $X$ un schéma noethérien de dimension pure, et $\mathcal{C}(X)$ l'ensemble des composantes irréductibles de $X$. On définit le cycle fondamental de $X$ comme la somme formelle

$$
[X]=\sum_{X^{\prime} \in \mathcal{C}(X)} \ell_{\mathcal{O}_{X, X^{\prime}}}\left(\mathcal{O}_{X, X^{\prime}}\right) X^{\prime}
$$

De plus, l'entier $\ell_{\mathcal{O}_{X, X^{\prime}}}\left(\mathcal{O}_{X, X^{\prime}}\right)$ est appelé la multiplicité de la composante irréductible $X^{\prime} \in$ $\mathcal{C}(X)$ dans $X$.

Soient $V$ un espace vectoriel de rang fini sur un corps $k$, et $\mathbb{P}(V)$ l'espace projectif associé à $V$. Dans la Définition 2.1, si $X$ est un sous-schéma fermé de dimension pure de $\mathbb{P}(V)$, on définit le degré du cycle $[X]$ comme

$$
\sum_{X^{\prime} \in \mathcal{C}(X)} \ell_{\mathcal{O}_{X, X^{\prime}}}\left(\mathcal{O}_{X, X^{\prime}}\right) \operatorname{deg}_{\mathcal{O}_{V}(1)}\left(X^{\prime}\right)
$$

qui est égal à $\operatorname{deg}_{\mathcal{O}_{V}(1)}(X)$. Dans la suite, on désgine par $\operatorname{deg}(X)$ le degré $\operatorname{deg}_{\mathcal{O}_{V}(1)}(X)$ pour simplifier les notations.

Maintenant on donne la construction précise de la variété de Cayley d'un sous-schéma fermé de dimension pure $X$ de $\mathbb{P}(V)$. Certaines idées proviennent de [11, §3.2.B], où l'on considère un plongement dans la grassmannienne. La variété de Caylay paramétrise les sousschémas linéaires de dimension $n-\operatorname{dim}(X)-1 \operatorname{de} \mathbb{P}(V)$ dont l'intersection avec $X$ est non vide.

Soit $\check{G}=\operatorname{Gr}\left(d+1, V^{\vee}\right)$ la grassmannienne qui classifie les quotients de rang $d+1$ de $V^{\vee}$ (ou encore les sous-espaces de rang $d+1$ de $V$ ), où $V^{\vee}$ est l'espace dual de $V$. Par le plongement de Plücker $\check{G} \rightarrow \mathbb{P}\left(\bigwedge^{d+1} V^{\vee}\right)$, l'algèbre de coordonnées $B(\check{G})=\bigoplus_{D \geq 0} B_{D}(\check{G})$ de $\check{G}$ est une algèbre quotient homogène de l'algèbre $\bigoplus_{D \geq 0} \operatorname{Sym}^{D}\left(\bigwedge^{d+1} V^{\vee}\right)$. Pour expliquer le rôle de la coordonnée de Plücker, on considère la construction suivante: on désigne par

$$
\theta: V^{\vee} \otimes_{k}\left(\bigwedge^{d+1} V\right) \rightarrow \bigwedge^{d} V
$$

l'homomorphisme qui envoie $\xi \otimes\left(x_{0} \wedge \cdots \wedge x_{d}\right)$ sur

$$
\sum_{i=0}^{d}(-1)^{i} \xi\left(x_{i}\right) x_{0} \wedge \cdots \wedge x_{i-1} \wedge x_{i+1} \wedge \cdots \wedge x_{d} .
$$


Soit $\widetilde{\Gamma}$ la sous-variété de $\mathbb{P}(V) \times_{k} \mathbb{P}\left(\bigwedge^{d+1} V^{\vee}\right)$ qui classifie les point $(\xi, \alpha)$ tels que $\theta(\xi \otimes$ $\alpha)=0$.

Soient

$$
p^{\prime}: \mathbb{P}(V) \times_{k} \mathbb{P}\left(\bigwedge^{d+1} V^{\vee}\right) \rightarrow \mathbb{P}(V)
$$

et

$$
q^{\prime}: \mathbb{P}(V) \times_{k} \mathbb{P}\left(\bigwedge^{d+1} V^{\vee}\right) \rightarrow \mathbb{P}\left(\bigwedge^{d+1} V^{\vee}\right)
$$

les deux projections canoniques, et $v: \widetilde{\Gamma} \rightarrow \mathbb{P}(V) \times_{k} \mathbb{P}\left(\bigwedge^{d+1} V^{\vee}\right)$ le plongement canonique. On définit

$$
p=p^{\prime} \circ v: \widetilde{\Gamma} \rightarrow \mathbb{P}(V) \text { et } q=q^{\prime} \circ v: \widetilde{\Gamma} \rightarrow \mathbb{P}\left(\bigwedge^{d+1} V^{\vee}\right) .
$$

Alors on a un diagram commutatif comme suit

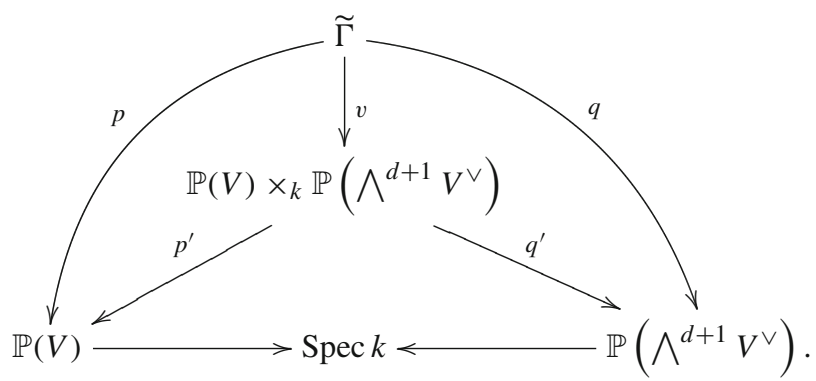

Avce la construction au-dessus, on a la proposition suivante, qui est une généralisation de [4, Proposition 3.4].

Proposition 2.2 Soit $X$ un sous-schéma fermé de dimension pure de $\mathbb{P}(V)$, qui est de dimension $d$. On suppose que $[X]=\sum_{i \in I} m_{i} X_{i}$ est le cycle fondamental de $X$. Alors $q_{*}\left(p^{*}[X]\right)$ est un diviseur sur $\mathbb{P}\left(\bigwedge^{d+1} V^{\vee}\right)$. De plus, ce diviseur est de la forme de $\sum_{i \in I} m_{i} \tilde{X}_{i}^{\prime}$, où chaque $\tilde{X}_{i}^{\prime}$ est une hypersurface intègre de degré $\operatorname{deg}\left(X_{i}\right)$ de $\mathbb{P}\left(\bigwedge^{d+1} V^{\vee}\right)$, et les $\tilde{X}_{i}^{\prime}$ sont distincts.

Proof D'abord, on considère le cas où $X$ est un schéma intègre. Dans ce cas-là, la démonstration suivante est inspirée par [4, Proposition 3.3]. La sous-variété d'incidence $\widetilde{\Gamma}$ est une fibration sur $\mathbb{P}(V)$. Comme $X$ est intègre, l'inverse schématique $p^{-1}(X)$ est irréductible. On désigne par $Y=p^{-1}(X)$, considéré comme un sous-schéma fermé et intègre de $\widetilde{\Gamma}$. La projection $q$ est propre, donc l'image $Z=q(Y)$ est un sous-schéma fermé intègre de $\mathbb{P}\left(\bigwedge^{d+1} V^{\vee}\right)$.

Soit $\xi=\operatorname{Spec} K$ un point fermé arbitraire de $Z$, qui est correspondant au $(d+1)$-ième puissance extérieure d'un sous-espace de rang $d+1$ de $V$. La fibre $Y_{\xi}$ coïncide avec le sous-schéma de $X_{K}$ défini par l'annulation sur $V$ au sens de (1) en prolongant $k$ dans $K$. On prend garde que la dimension de $X_{K}$ est $d$. Donc $q$ envoie $Y$ dans $Z$ birationnellement et on $\operatorname{adim}(Z)=\operatorname{dim}(Y)=\operatorname{dim}\left(\mathbb{P}\left(\bigwedge^{d+1} V^{\vee}\right)\right)-1$. 
Afin de calculer le degré de $Z$ dans $\mathbb{P}\left(\bigwedge^{d+1} V^{\vee}\right)$, on considère l'égalité de la classe des cycles

$$
[Z]=q_{*}\left(p^{*}[X]\right)=\operatorname{deg}(X) \cdot q_{*}\left(p^{*}[U]\right),
$$

où $U$ est l'espace projectif associé à un espace quotient de dimension $d+1$ de $V$ arbitraire prolongé dans $\mathbb{P}\left(\bigwedge^{d+1} V^{\vee}\right)$ par le plongement de Plücker. On prend garde que $q_{*}\left(p^{*}[U]\right)$ est la classe première de Schubert dans $\mathbb{P}\left(\bigwedge^{d+1} V^{\vee}\right)$ (cf. [10, §14.7]), alors le degré de $Z$ est $\operatorname{deg}(X)$.

Dans la suite, on considère le cas de schémas généraux de dimension pure. Si $X$ est un sous-schéma fermé de dimension pure de $\mathbb{P}(V)$ avec le cycle fondamental dans l'énoncé, on a

$$
q_{*}\left(p^{*}[X]\right)=\sum_{i \in I} m_{i} q_{*}\left(p^{*}\left[X_{i}\right]\right)
$$

Soient $X_{i}$ et $X_{j}$ deux composantes irréductibles distinctes de $X$, qui sont considérées comme deux schémas intègres. Donc il existe un $\bar{k}$-point $P$ dans $\mathbb{P}(V)$, tel que $P \in X_{i}(\bar{k})$ mais $P \notin X_{j}(\bar{k})$. De plus, on obtient qu'il existe un sous-schéma $\bar{k}$-linéaire fermé qui intersecte $X_{i}$ en un sous-schéma non vide mais n'intersecte pas $X_{j}$. On en déduit $q_{*}\left(p^{*}\left[X_{i}\right]\right) \neq$ $q_{*}\left(p^{*}\left[X_{j}\right]\right)$ comme des cycles premiers.

Definition 2.3 Soit $X$ un sous-schéma fermé de dimension pure de $\mathbb{P}(V)$. On dit que le cycle déterminé dans la Proposition 2.2 est diviseur de Cayley de $X$. De plus, on dit que l'hypersurface de $\mathbb{P}\left(\bigwedge^{d+1} V^{\vee}\right)$ dont le cycle fondamental est celui déterminé dans la Proposition 2.2 est la variété de Cayley de $X$.

Remarque 2.4 Avec la construction dans la Proposition 2.2. Si on remplace $\mathbb{P}\left(\bigwedge^{d+1} V^{\vee}\right)$ par la grassmannienne $\operatorname{Gr}\left(d+1, V^{\vee}\right)$, on a presque le même résultat. Dans ce cas-là, le cycle sur $\operatorname{Gr}\left(d+1, V^{\vee}\right)$ obtenu par le sens similaire est appelé le diviseur de Chow, et l'hypersurface de $\operatorname{Gr}\left(d+1, V^{\vee}\right)$ obtenue par le même sens que celui dans la Définition 2.3 est appelée la variété de Chow, qui est de degré $\delta$ aussi. Voir [11, §3.2.B] pour plus détails sur l'approche.

\subsection{Le diviseur de Cayley sur un anneau de Dedekind}

Dans cette partie, on donnera une construction de diviseurs de Cayley sur un anneau de Dedekind. Certaines idées sont inspirées par [1, \$4.3.1].

Soient $A$ un anneau de Dedekind, $\mathcal{E}$ un fibré vectoriel de $\operatorname{rang} n+1 \operatorname{sur} \operatorname{Spec} A$, et $\mathcal{E}^{\vee}$ le fibré dual de $\mathcal{E}$. À tout schéma $L$ sur $\operatorname{Spec} A$, le foncteur grassmannien associe l'ensemble de modules quotients localement libres de $\mathcal{E}^{\vee} \otimes_{A} \mathcal{O}_{L}$ de rang $d+1 \operatorname{sur} \mathcal{O}_{L}$. On désigne par $\operatorname{Gr}\left(d+1, \mathcal{E}^{\vee}\right)$ le schéma qui représente ce foncteur grassmannien. En particulier, si $d=0$, on le désigne par $\mathbb{P}(\mathcal{E})$ pour des raisons de simplicité.

Dans la suite, on introduit le plongement de Plücker $\operatorname{Gr}\left(d+1, \mathcal{E}^{\vee}\right) \rightarrow \mathbb{P}\left(\bigwedge^{d+1} \mathcal{E}^{\vee}\right)$, et le sous-schéma d'incidence $\widetilde{\Gamma}$ de $\mathbb{P}(\mathcal{E}) \times_{\operatorname{Spec} A} \mathbb{P}\left(\bigwedge^{d+1} \mathcal{E}^{\vee}\right)$ sur Spec $A$. Pour toute $A$ algèbre $k$ qui est un corps, le plongement de Plücker envoie un point de $\operatorname{Gr}\left(d+1, \mathcal{E}_{k}^{\vee}\right)$ dans 
$\mathbb{P}\left(\bigwedge^{d+1} \mathcal{E}_{k}^{\vee}\right)$ par (1). De plus, les points de $\widetilde{\Gamma}$ à valeur dans $k$ sont les couples

$$
(\xi, \alpha) \in \mathbb{P}\left(\mathcal{E}_{k}\right)(k) \times \mathbb{P}\left(\bigwedge^{d+1} \mathcal{E}_{k}^{\vee}\right)(k)
$$

satisfaisant $\theta(\xi \otimes \alpha)=0$, où $\theta$ est défini dans (1).

Soient

$$
\tilde{p}^{\prime}: \mathbb{P}(\mathcal{E}) \times_{\operatorname{Spec} A} \mathbb{P}\left(\bigwedge^{d+1} \mathcal{E}^{\vee}\right) \rightarrow \mathbb{P}(\mathcal{E})
$$

et

$$
\tilde{q}^{\prime}: \mathbb{P}(\mathcal{E}) \times_{\operatorname{Spec} A} \mathbb{P}\left(\bigwedge^{d+1} \mathcal{E}^{\vee}\right) \rightarrow \mathbb{P}\left(\bigwedge^{d+1} \mathcal{E}^{\vee}\right)
$$

les deux projections canoniques. et $\widetilde{v}: \widetilde{\Gamma} \rightarrow \mathbb{P}(\mathcal{E}) \times_{\operatorname{Spec} A} \mathbb{P}\left(\bigwedge^{d+1} \mathcal{E}^{\vee}\right)$ le plongement canonique. On définit

$$
\widetilde{p}=\tilde{p}^{\prime} \circ \widetilde{v}: \widetilde{\Gamma} \rightarrow \mathbb{P}(\mathcal{E}) \text { et } \widetilde{q}=\widetilde{q}^{\prime} \circ \widetilde{v}: \widetilde{\Gamma} \rightarrow \mathbb{P}\left(\bigwedge^{d+1} \mathcal{E}^{\vee}\right)
$$

Alors on a le diagramm commutatif

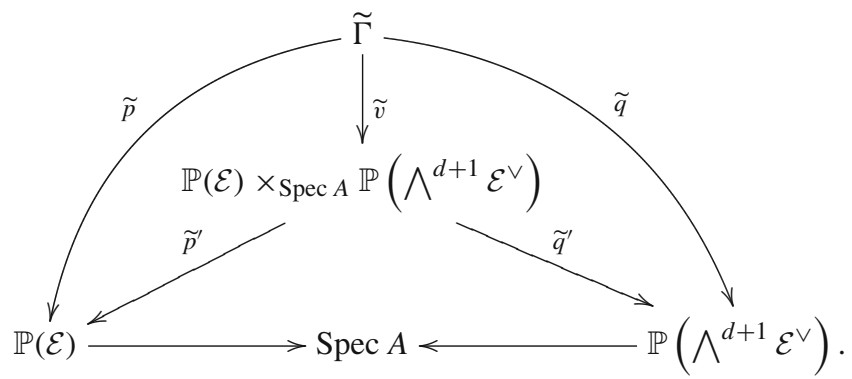

On a la proposition suivante concernant le cycle $\widetilde{q}_{*}\left(\widetilde{p}^{*}[\mathscr{X}]\right)$, dont la démonstration est même que celle de [1, Lemma 4.3.1].

Proposition 2.5 Soit $\mathscr{X}$ un sous-schéma fermé de dimension pure de $\mathbb{P}(\mathcal{E})$. Si $\mathscr{X}$ est plat sur $\operatorname{Spec} A$ (resp. plat sur Spec A et irréductible), $\widetilde{q}_{*}\left(\widetilde{p}^{*}[\mathscr{X}]\right) \rightarrow \operatorname{Spec} A$ l'est aussi.

Proof D'abord, on suppose que $\mathscr{X}$ est un schéma intègre. Un schéma intègre sur Spec $A$ est plat si et seulement si son point générique se trouve au-dessus du point générique Spec $K$ de Spec $A$. Comme le morphisme $\widetilde{p}: \widetilde{\Gamma} \rightarrow \mathbb{P}(\mathcal{E})$ est lisse à fibres géométriquement connexes, le cycle $\widetilde{p}^{*}(\mathscr{X})$ est irréductible aussi. Son point générique se trouve au-dessus de celui de $\mathscr{X}$, et se trouve au-dessus de $\operatorname{Spec} K$.

On désigne par $m W$ le cycle $\widetilde{q}_{*}\left(\widetilde{p}^{*}[\mathscr{X}]\right)$, où $m$ est un entier positif. Dans ce cas-là, $W$ est plat sur Spec $A$. L'entier $m$ sera zéro si $W$ est de codimension plus grande que ou égale à 1 dans $\tilde{p}^{*}(\mathscr{X})($ cf. $[10, \S 1.4])$, sinon $m$ s'indentifie au degré du corps des fonctions rationnelles de $\widetilde{p}^{*}(\mathscr{X})$ sur celui de $W$. Comme l'image directe propre commute avec l'image inverse (cf. [10, Proposition 1.7 et $\$ 20.1]$ ), on est capacité de calculer $m$ en appliquant le changement de base Spec $K \rightarrow \operatorname{Spec} A$. Alors on réduit le problème au cas où l'annear de base est un corps, qui est démontré dans la Proposition 2.2, voir [4, Proposition 3.4] aussi. 
Si $\mathscr{X}$ n'est pas intègre, soit $[\mathscr{X}]=\sum_{i \in I} m_{i}\left[\mathscr{X}_{i}\right]$ le cycle fondamental de $\mathscr{X}$, alors on a

$$
\widetilde{q}_{*}\left(\widetilde{p}^{*}[\mathscr{X}]\right)=\sum_{i \in I} m_{i} \widetilde{q}_{*}\left(\widetilde{p}^{*}\left[\mathscr{X}_{i}\right]\right) .
$$

Donc on a le résultat en appliquant l'argument ci-dessus composante par composante.

Definition 2.6 Soit $\mathscr{X}$ un sous-schéma fermé de $\mathbb{P}(\mathcal{E})$ sur Spec $A$. On dit que le cycle $\widetilde{q}_{*}\left(\widetilde{p}^{*}[\mathscr{X}]\right)$ est le diviseur de Cayley de $\mathscr{X} \operatorname{sur} \mathbb{P}\left(\bigwedge^{d+1} \mathcal{E}^{\vee}\right)$.

La proposition suivante est autour de la commutativité de la construction du diviseur de Cayley et certains changements de base, qui est énoncé dans [1, §4.3.2 (i)]. On va donner une démonstration détaillée de l'assertion pour le cas d'anneau de Dedekind.

Proposition 2.7 Avec toutes les notations et la constructions dans cette partie. Soit $\mathscr{X}$ un sous-schéma fermé de dimension pure de $\mathbb{P}(\mathcal{E})$ sur $\mathrm{Spec} A$, où A est un anneau de Dedekind. Soit $T$ un schéma noethérien régulier qui satisfait l'une des deux condition suivantes:

(i) le changement de base $T \rightarrow$ Spec A est plat;

(ii) le schéma $T$ est un point fermé dans le changement de base $T \rightarrow \operatorname{Spec} A$, et $\mathscr{X} \times \operatorname{Spec} A$ $T \rightarrow T$ et $\mathscr{X} \rightarrow$ Spec $A$ ont la même dimension relative.

Alors on a

$$
\left(\widetilde{q}_{T}\right)_{*}\left(\left(\widetilde{p}_{T}\right)^{*}\left[\mathscr{X}_{T}\right]\right)=\left(\widetilde{q}_{*}\left(\tilde{p}^{*}[\mathscr{X}]\right)\right)_{T} .
$$

Autrement dit, la construction du diviseur de Cayley commute au changement de base satisfaisant les condtions ci-dessus.

Proof Par la construction dans l'énoncé, on a le diagram commutatif

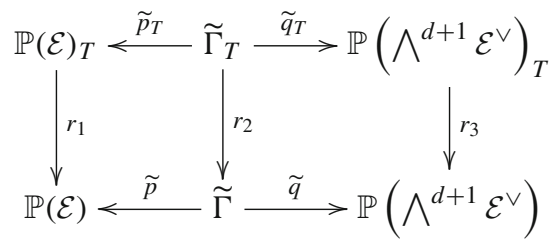

induit par le changement de base $T \rightarrow \operatorname{Spec} A$.

(i) Si le changement de base $T \rightarrow \operatorname{Spec} A$ est plat, les morphismes $r_{1}, r_{2}$ et $r_{3}$ sont plats aussi. Par définition, on a $\tilde{p} \circ r_{2}=r_{1} \circ \widetilde{p}_{T}$, d'où l'on a

$$
r_{2}^{*}\left(\tilde{p}^{*}[\mathscr{X}]\right)=\tilde{p}_{T}^{*}\left(r_{1}^{*}[\mathscr{X}]\right)
$$

par [10, Lemma 1.7.1] couplé avec [10, §20.1].

On désigne $[\mathscr{Y}]=\widetilde{p}^{*}[\mathscr{X}]$ pour simplifier. Car le morphisme $\widetilde{q}$ est propre, on a

$$
r_{3}^{*}\left(\widetilde{q}_{*}[\mathscr{Y}]\right)=\left(\widetilde{q}_{T}\right)_{*}\left(r_{2}^{*}[\mathscr{Y}]\right)
$$

d'après [10, Proposition 1.7] couplé avec [10, §20.1], qui termine la démonstration.

(ii) Maintenant on considère le cas satisfaisant la condition (ii). Dans ce cas-là, $T$ est un diviseur de Cartier sur $\operatorname{Spec} A$. Alors on peut identifier $\mathbb{P}(\mathcal{E})_{T}$ (resp. $\widetilde{\Gamma}_{T}$ et $\left.\mathbb{P}\left(\wedge^{d+1} \mathcal{E}^{\vee}\right)_{T}\right)$ à un sous-schéma fermé de codimension 1 de $\mathbb{P}(\mathcal{E})$ (resp. $\widetilde{\Gamma}$ et $\mathbb{P}\left(\bigwedge^{d+1} \mathcal{E}^{\vee}\right)$ ), à savoir la fibre de $\mathbb{P}(\mathcal{E})\left(\right.$ resp. $\widetilde{\Gamma}$ et $\mathbb{P}\left(\bigwedge^{d+1} \mathcal{E}^{\vee}\right)$ ) au-dessus de l'image de $T \rightarrow$ Spec $A$. Alors les morphismes $r_{1}, r_{2}$ et $r_{3}$ sont des immersions fermées. 
Avant tout, on suppose que $\mathscr{X}$ est intègre. D'abord, on démontrera $\mathscr{X} \nsubseteq \mathbb{P}(\mathcal{E})_{T}$ comme des sous-schémas fermé de $\mathbb{P}(\mathcal{E})$. Si $\mathscr{X} \subseteq \mathbb{P}(\mathcal{E})_{T}$, alors $\mathscr{X}_{T}$ a la même dimension que celle de $\mathscr{X}$. La dimension de $\mathbb{P}(\mathcal{E})_{T}$ et 1 plus petite que celle de $\mathbb{P}(\mathcal{E})$, donc on a une contradiction à partir de la condition sur la dimension relative dans (ii).

Alors on a $\mathscr{X} \nsubseteq \mathbb{P}(\mathcal{E})_{T}$. Par la construction dans [10, Definition 2.3, Remark 2.3] couplé avec $[10, \S 20.1]$, on a

$$
[\mathscr{X}] \cdot\left[\mathbb{P}(\mathcal{E})_{T}\right]=\left[\mathscr{X} \cap \mathbb{P}(\mathcal{E})_{T}\right]=\left[r_{1}^{-1}(\mathscr{X})\right]=r_{1}^{*}[\mathscr{X}]
$$

comme des cycles sur $\mathbb{P}(\mathcal{E})_{T}$.

De plus, les morphismes $\tilde{p}$ et $\widetilde{p}_{T}$ sont lisses et ont les fibres géométriquement connexes,

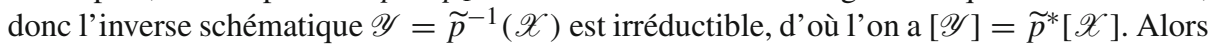
par le même argument que celui ci-dessus, on a

$$
r_{2}^{*}[\mathscr{Y}]=[\mathscr{Y}] \cdot\left[\widetilde{\Gamma}_{T}\right] .
$$

comme des cycles sur $\widetilde{\Gamma}_{T}$. De plus, par [10, Definition 2.3, Remark 2.3] encore couplé avec $[10, \S 20.1]$, on a

$$
[\mathscr{Y}] \cdot\left[\widetilde{\Gamma}_{T}\right]=\left[\tilde{p}^{-1}(\mathscr{X}) \cap \widetilde{p}_{T}^{-1}\left(\mathbb{P}(\mathcal{E})_{T}\right)\right]=\left[\widetilde{p}^{-1}\left(\mathscr{X} \cap \mathbb{P}(\mathcal{E})_{T}\right)\right]=\widetilde{p}_{T}^{*}\left(r_{1}^{*}[\mathscr{X}]\right)
$$

comme des cycles sur $\widetilde{\Gamma}_{T}$.

Par les arugments ci-dessus, on obtient

$$
r_{2}^{*}\left(\widetilde{p}^{*}[\mathscr{X}]\right)=\widetilde{p}_{T}^{*}\left(r_{1}^{*}[\mathscr{X}]\right),
$$

qui signifie

$$
\left(\widetilde{p}^{*}[\mathscr{X}]\right)_{T}=\widetilde{p}_{T}^{*}\left(\left[\mathscr{X}_{T}\right]\right) .
$$

Dans la suite, on a $\mathscr{Y} \nsubseteq \widetilde{\Gamma}_{T}$, considérés commes des sous-schémas fermés de $\widetilde{\Gamma}$ par la condition sur les dimensions relatives dans (ii) aussi. Car le morphism $\widetilde{q}$ est propre, on a

$$
\widetilde{q}_{*}\left([\mathscr{Y}] \cdot\left[\widetilde{\Gamma}_{T}\right]\right)=\widetilde{q}_{*}[\mathscr{Y}] \cdot\left[\mathbb{P}\left(\bigwedge^{d+1} \mathcal{E}^{\vee}\right)_{T}\right]
$$

par la formule de projection de cycles dans la théorie de l'intersection (cf. [21, Chap. 5, C), $\S 7,(10)])$, d'où l'on a

$$
\left(\widetilde{q}_{T}\right)_{*}\left[\mathscr{Y}_{T}\right]=\left(\widetilde{q}_{*}[\mathscr{Y}]\right)_{T}
$$

Si $\mathscr{X}$ n'est pas intègre, on a le résultat en appliquant l'argument ci-dessus composante par composante.

Remarque 2.8 Dans [1, §4.3.2 (i)], on remplace Spec $A$ dans la Proposition 2.7 par un schéma noethérien régulier $T$, et on considère un plongement fermé régulier de $T^{\prime}$ dans $T$. Dans ce cas-là, la formation de la variété de Chow encore commute au changement de base $T^{\prime} \rightarrow T$ si on suppose la même condition sur la dimension relative que celle dans la Proposition 2.7 (ii).

\section{Hauteurs d'un schéma projectif}

Dans cette section, on introduira certaines fonctions hauteur et les comparera. 


\subsection{Préliminaires}

Afin d'introduire des fonctions hauteur, d'abord on introduit certaines notions de base de la théorie algébrique des nombres. On utilisera ces notions dans tout l'article sauf mention contraire.

Soient $K$ un corps de nombres, et $\mathcal{O}_{K}$ l'anneau des entiers de $K$. Dans tout l'article, on désigne par $M_{K, f}$ l'ensemble des places finies de $K$, par $M_{K, \infty}$ l'ensemble des places infinies de $K$ et par $M_{K}$ l'ensemble des places de $K$.

\section{Fibré vectoriel normé}

On appelle fibré vectoriel normé sur $\operatorname{Spec} \mathcal{O}_{K}$ toute donnée $\overline{\mathcal{E}}=(\mathcal{E}, h)$, où:

1. $\mathcal{E}$ est un $\mathcal{O}_{K}$-module projectif de rang fini;

2. $h=\left(\|\cdot\|_{v}\right)_{v \in M_{K, \infty}}$ est une famille de normes, où $\|\cdot\|_{v}$ est une norme sur $\mathcal{E} \otimes_{\mathcal{O}_{K, v}} \mathbb{C}$ qui est invariante sous l'action du groupe $\mathrm{Gal}\left(\mathbb{C} / K_{v}\right)$.

Le rang de $\overline{\mathcal{E}}$ est défini comme celui de $\mathcal{E}$. Si toutes les normes dans $h$ sont hermitiennes, on dit que $\overline{\mathcal{E}}$ est un fibré vectoriel hermitien sur $\operatorname{Spec} \mathcal{O}_{K}$. Si le rang de $\overline{\mathcal{E}}$ est 1 , on dit que $\overline{\mathcal{E}}$ est un fibré en driotes hermitien $\operatorname{sur} \operatorname{Spec} \mathcal{O}_{K}$.

Soient $\bar{L}=\left(L,\left(\|\cdot\|_{v}\right)_{v \in M_{K, \infty}}\right)$ un fibré en droites hermitien $\operatorname{sur} \operatorname{Spec} \mathcal{O}_{K}$, et $s \in L \otimes \mathcal{O}_{K} K$ un élément non-nul. Pour une place $v \in M_{K}, f$, si $\mathbb{Q}_{v}$ est le corps $p$-adique, on définit $|a|_{v}=\left|N_{K_{v} / \mathbb{Q}_{v}}(a)\right|_{p}^{1 /\left[K_{v}: \mathbb{Q}_{v}\right]}$, où $|\cdot|_{p}$ est la valeur $p$-adique. De plus, on définit la norme $\|s\|_{v}=\inf \left\{|a|_{v} \mid a \in K_{v}^{\times}, a^{-1} s \in L \otimes_{\mathcal{O}_{K}} \widehat{\mathcal{O}}_{K, v}\right\}$ donnée par le modèle.

\section{Fonction hauteur}

Soient $\overline{\mathcal{E}}$ un fibré vectoriel hermitien de rang $n+1 \operatorname{sur} \operatorname{Spec} \mathcal{O}_{K}$, et $\mathcal{E}_{K}=\mathcal{E} \otimes_{\mathcal{O}_{K}} K$. On désigne par Chow ${ }_{d, \delta}^{n}(K)$ l'ensemble des sous-schémas fermés de $\mathbb{P}\left(\mathcal{E}_{K}\right)$, qui sont de dimension pure $d$ et de degré $\delta$ plongés dans $\mathbb{P}\left(\mathcal{E}_{K}\right)$. Soit $\overline{\mathcal{L}}=\left(\mathcal{L},\left(\|\cdot\|_{v}\right)_{v \in M_{K, \infty}}\right)$ un fibré en droites ample arithmétique hermitien sur $\mathbb{P}(\mathcal{E})$. Alors la hauteur d'un schéma projectif par rapport au fibré en droites hermitien $\overline{\mathcal{L}}$ est une fonction

$$
h_{\overline{\mathcal{L}}}: \operatorname{Chow}_{d, \delta}^{n}(K) \rightarrow \mathbb{R}
$$

qui mesure la complexité arithmétique d'un $K$-schéma projectif.

Plusieurs fonctions hauteur de schémas arithmétiques seront utilisées dans cet article. Soient $X$ un sous-schéma fermé de $\mathbb{P}\left(\mathcal{E}_{K}\right)$ de dimension pure, et $\mathscr{X}$ l'adhérence schématique de $X$ dans $\mathbb{P}(\mathcal{E})$. D'abord on introduira une hauteur de $\mathscr{X}$ pour le cas général. Au cas où $X$ est une hypersurface, on a quelques propriétés spéciales.

\subsection{Hauteurs d'un schéma projectif de dimension pure}

D'abord, on définit une fonction hauteur introduite par Faltings dans [8, Definition 2.5] par la théorie de l'intersection arithmétrique. La théorie de l'intersection arithmétique est développée par Gillet et Soulé dans [12], voir [22] pour une introduction systématique de cette théorie.

Definition 3.1 [Hauteur arakelovienne] Soient $\overline{\mathcal{E}}$ un fibré vectoriel hermitien de rang $n+1$ sur Spec $\mathcal{O}_{K}$, et $\overline{\mathcal{L}}$ un fibré en droites hermitien sur $\mathbb{P}(\mathcal{E})$. Soient $X$ un sous-schéma fermé 
de dimension pure $d$ de $\mathbb{P}\left(\mathcal{E}_{K}\right)$, et $\mathscr{X}$ l'adhérence schématique de $X$ dans $\mathbb{P}(\mathcal{E})$. La hauteur arakelovienne de $X$ est définie comme le nombre de l'intersection arithmétique

$$
\frac{1}{[K: \mathbb{Q}]} \widehat{\operatorname{deg}}\left(\widehat{c}_{1}(\overline{\mathcal{L}})^{d+1} \cdot[\mathscr{X}]\right),
$$

où $\widehat{c}_{1}(\overline{\mathcal{L}})$ est la première classe de Chern arithmétique de $\overline{\mathcal{L}}$. Cette hauteur est notée comme $h_{\overline{\mathcal{L}}}(X)$ ou $h_{\overline{\mathcal{L}}}(\mathscr{X})$.

\subsection{Hauteur d'une hypersurface projective}

Soient $\overline{\mathcal{E}}$ un fibré vectoriel hermitien de rang $n+1 \operatorname{sur} \operatorname{Spec} \mathcal{O}_{K}$, et $f\left(T_{0}, \ldots, T_{n}\right)$ un polynôme homogène à coefficients dans $K$ de degré $\delta$, alors

$$
X=\operatorname{Proj}\left(K\left[T_{0}, \ldots, T_{n}\right] /\left(f\left(T_{0}, \ldots, T_{n}\right)\right)\right)
$$

est un sous-schéma fermé de $\mathbb{P}\left(\mathcal{E}_{K}\right)$ de dimension $n-1$. Il est en fait une hypersurface de $\mathbb{P}\left(\mathcal{E}_{K}\right)$ de degré $\delta$ (cf. [15, Proposition 7.6, Chap. I]). Dans cette partie, on discutera des hauteurs d'une hypersurface dans $\mathbb{P}\left(\mathcal{E}_{K}\right)$.

Pour tout $v \in M_{K, \infty}$, on désigne par $|\cdot|_{v}$ la valeur absolue à la place $v$ qui satisfait $|a|_{v}=\left|N_{K_{v} / \mathbb{Q}_{v}}(a)\right|^{1 /\left[\hat{K}_{v}: \mathbb{Q}_{v}\right]}$, où $|\cdot|$ est la valeur absolue usuelle sur $\mathbb{R}$ ou $\mathbb{C}$.

Definition 3.2 (Hauteur classique) Soit

$$
f\left(T_{0}, \ldots, T_{n}\right)=\sum_{\left(i_{0}, \ldots, i_{n}\right) \in \mathbb{N}^{n+1}} a_{i_{0}, \ldots, i_{n}} T_{0}^{i_{0}} \cdots T_{n}^{i_{n}}
$$

un polynôme non-nul à coefficients dans $K$. La hauteur classique $h(f)$ du polynôme homogène $f$ est définie comme

$$
h(f)=\sum_{v \in M_{K}} \frac{\left[K_{v}: \mathbb{Q}_{v}\right]}{[K: \mathbb{Q}]} \log \max _{\left(i_{0}, \ldots, i_{n}\right) \in \mathbb{N}^{n+1}}\left\{\left|a_{i_{0}, \ldots, i_{n}}\right|_{v}\right\} \in \mathbb{R}_{+} .
$$

De plus, si $f$ est homogène et $X$ est l'hypersurface de $\mathbb{P}\left(\mathcal{E}_{K}\right)$ définie par $f$, on définit $h(X)=h(f)$ comme la hauteur classique de l'hypersurface $X$.

La hauteur introduite dans la Définition 3.2 est invariante sous l'extension finie de corps de nombres.

Afin d'introduire une autre fonction hauteur, on introduit la mesure de Mahler.

Definition 3.3 (Mesure de Mahler) Soit $f\left(T_{1}, \ldots, T_{n}\right) \in \mathbb{C}\left[T_{1}, \ldots, T_{n}\right]$ un polynôme. On définit la mesure de Mahler du polynôme $f\left(T_{1}, \ldots, T_{n}\right)$ comme

$$
M(f)=\exp \left(\int_{[0,1]^{n}} \log \left|f\left(e^{2 \pi i t_{1}}, \ldots, e^{2 \pi i t_{n}}\right)\right| d t_{1} \cdots d t_{n}\right),
$$

où $|\cdot|$ est la valeur absolue usuelle sur $\mathbb{C}$.

Pour un corps de nombres $K$, soient $f\left(T_{1}, \ldots, T_{n}\right) \in K\left[T_{1}, \ldots, T_{n}\right]$ et $v: K \hookrightarrow \mathbb{C}$ un plongement. On définit

$$
M(v(f))=\exp \left(\int_{[0,1]^{n}} \log \left|v(f)\left(e^{2 \pi i t_{1}}, \ldots, e^{2 \pi i t_{n}}\right)\right| d t_{1} \cdots d t_{n}\right)
$$

comme la mesure de Mahler du polynôme $f$ par rapport au plongement $v$. 
On va introduire la fonction hauteur ci-dessous, qui est originaire de [19, Définition 1.10].

Definition 3.4 (Hauteur de Philippon) Soit $X$ une hypersurface de $\mathbb{P}\left(\mathcal{E}_{K}\right)$ définie par le polynôme homogène

$$
f\left(T_{0}, \ldots, T_{n}\right)=\sum_{\substack{\left(i_{0}, \ldots, i_{n}\right) \in \mathbb{N}^{n+1} \\ i_{0}+\cdots+i_{n}=\delta}} a_{i_{0}, \ldots, i_{n}} T_{0}^{i_{0}} \cdots T_{n}^{i_{n}},
$$

la hauteur de Philippon de $X$ est définie comme

$$
h_{P h}(X):=\sum_{v \in M_{K, f}} \frac{\left[K_{v}: \mathbb{Q}_{v}\right]}{[K: \mathbb{Q}]} \log \|f\|_{v}+\frac{1}{[K: \mathbb{Q}]} \sum_{v \in M_{K, \infty}} \log M(v(f)),
$$

où l'on définit

$$
\|f\|_{v}=\max _{\substack{\left(i_{0}, \ldots, i_{n}\right) \in \mathbb{N}^{n+1} \\ i_{0}+\ldots+i_{n}=\delta}}\left\{\left|a_{i_{0}, \ldots, i_{n}}\right|_{v}\right\}
$$

pour tout $v \in M_{K, f}$, et $M(v(f))$ est la mesure de Mahler de $f$ par rapport à la place $v \in M_{K, \infty}$ définie par (2) dans la Définition 3.3.

Soient $\overline{\mathcal{E}}$ un fibré vectoriel hermitien $\operatorname{sur} \operatorname{Spec} \mathcal{O}_{K}$, et $s \in H^{0}\left(\mathbb{P}\left(\mathcal{E}_{K}\right), \mathcal{O}_{\mathbb{P}\left(\mathcal{E}_{K}\right)}(\delta)\right)$ une section globale non-nulle. Pour toute place infinie $v \in M_{K, \infty}$ fixée, on désigne par $\|\cdot\|_{v, \mathrm{FS}}$ la métrique de Fubini-Study sur $\mathbb{P}\left(\mathcal{E}_{K, v}\right)(\mathbb{C})$ par rapport à la place infinie $v$. De plus, on définit

$$
\|s\|_{v, \infty}=\sup _{x \in \mathbb{P}\left(\mathcal{E}_{K, v}\right)(\mathbb{C})}\|s(x)\|_{v, \mathrm{FS}}=\sup _{\|x\|_{v}=1}\|s(x)\|_{v, \mathrm{FS}}
$$

Soient $U\left(\mathcal{E}_{K, v},\|\cdot\|_{v}\right)$ le groupe unitaire qui agit $\operatorname{sur} \mathcal{E}_{K, v}$, et $d v(x)$ une mesure $U\left(\mathcal{E}_{K, v},\|\cdot\|_{v}\right)$ invariante unique probabiliste $\operatorname{sur} \mathbb{P}\left(\mathcal{E}_{K, v}\right)(\mathbb{C})$, ce qui signifie

$$
\int_{\mathbb{P}\left(\mathcal{E}_{K, v}\right)(\mathbb{C})} d v(x)=1 .
$$

En suite, on définit

$$
\|s\|_{v, 0}=\exp \left(\int_{\mathbb{P}\left(\mathcal{E}_{K, v}\right)(\mathbb{C})} \log \|s(x)\|_{v, \mathrm{FS}} d v(x)\right) .
$$

Pour tout nombre réel strictement positif $p$, on définit

$$
\|s\|_{v, p}=\left(\int_{\mathbb{P}\left(\mathcal{E}_{K, v}\right)(\mathbb{C})}\|s(x)\|_{v, \mathrm{FS}}^{p} d v(x)\right)^{1 / p} .
$$

Avec les norme (4), (5) et (6) sur l'espace $H^{0}\left(\mathbb{P}\left(\mathcal{E}_{K}\right), \mathcal{O}_{\mathbb{P}\left(\mathcal{E}_{K}\right)}(\delta)\right)$, on définit la fonction hauteur suivante.

Definition 3.5 ( $p$-hauteur) Soit $s \in H^{0}\left(\mathbb{P}\left(\mathcal{E}_{K}\right), \mathcal{O}_{\mathbb{P}\left(\mathcal{E}_{K}\right)}(\delta)\right)$ non-nulle. On définit la $p$ hauteur de l'hypersurface $X$ de $\mathbb{P}\left(\mathcal{E}_{K}\right)$ définie par la section globale $s$ comme

$$
h_{p}(X)=\sum_{v \in M_{K, f}} \frac{\left[K_{v}: \mathbb{Q}_{v}\right]}{[K: \mathbb{Q}]} \log \|s\|_{v}+\sum_{v \in M_{K, \infty}} \frac{\left[K_{v}: \mathbb{Q}_{v}\right]}{[K: \mathbb{Q}]} \log \|s\|_{v, p},
$$

où $\|\cdot\|_{v}$ est la même que (3) pour $v \in M_{K, f}$ lorsque $s$ est considéré comme un polynôme homogène, et la norme $\|\cdot\|_{v, p}$ est définie dans les égalités (4), (5) et (6) pour les $p \in[0,+\infty]$. 


\section{Hauteur de la variété de Cayley}

On a déjà défini la variété de Cayley dans §2. Dans cette partie, on va étudier la hauteur de variété de Cayley.

On considère le fibré vectoriel hermitien

$$
\overline{\mathcal{E}}=\left(\mathcal{O}_{K}^{\oplus(n+1)},\left(\|\cdot\|_{v}\right)_{v \in M_{K, \infty}}\right)
$$

sur $\operatorname{Spec} \mathcal{O}_{K}$, qui est muni des $\ell^{2}$-normes définies suivantes: pour tout plongement $v: K \hookrightarrow$ $\mathbb{C}$, la norme $\|\cdot\|_{v}$ envoie le point $\left(x_{0}, \ldots, x_{n}\right)$ sur $\sqrt{\left|v\left(x_{0}\right)\right|^{2}+\cdots+\left|v\left(x_{n}\right)\right|^{2}}$.

Soit $X$ un sous-schéma de dimension pure de dimension $d$ et degré $\delta$ dans $\mathbb{P}\left(\mathcal{E}_{K}\right)$ avec le fibré hermitien $\overline{\mathcal{E}}$ sur $\operatorname{Spec} \mathcal{O}_{K}$ dans (7). On désigne par $\widetilde{h}_{0}(X)$ la 0 -hauteur de la variété de Cayley de $X$ définie dans la Définition 3.5. D'après [1, Theorem 4.3.8], on a

$$
\widetilde{h}_{0}(X)=h \overline{\mathcal{O}(1)}(X)-\frac{1}{2} \delta \mathcal{H}_{N},
$$

où $N=\operatorname{rg}\left(\bigwedge^{d+1} \mathcal{E}_{K}\right)-1=\left(\begin{array}{c}n+1 \\ d+1\end{array}\right)-1, \mathcal{H}_{N}=1+\frac{1}{2}+\cdots+\frac{1}{N}$, et $\overline{\mathcal{O}(1)}$ est muni des métriques de Fubini-Study à partir de $\overline{\mathcal{E}}$ ci-dessus.

\section{Sur la comparaison des hauteurs}

Dans cette partie, on comparera certaines hauteurs utiles de variétés arithmétiques. Soient $\overline{\mathcal{E}}$ le fibré hermitien défini dans (7), et $s \in H^{0}\left(\mathbb{P}\left(\mathcal{E}_{K}\right), \mathcal{O}_{\mathbb{P}\left(\mathcal{E}_{K}\right)}(\delta)\right)$ une section globale non-nulle. On considère une telle section comme un polynôme homogène de degré $\delta$ dans $K\left[T_{0}, \ldots, T_{n}\right]$. D'après $[20$, Théorème 1$]$, on a

$$
0 \leq \log M(v(s))-\log \|s\|_{v, 0} \leq 4 \delta \log (n+1)
$$

pour toute place $v \in M_{K, \infty}$, où $M(v(s))$ est la mesure de Mahler de la section $s$ considérée comme un polynôme homogène de degré $\delta$ par rapport à cette place, voir la Définition 3.3 et (2) pour la définition.

Soit $X$ une hypersurface de $\mathbb{P}\left(\mathcal{E}_{K}\right)$ de degré $\delta$, alors on en déduit

$$
0 \leq h_{P h}(X)-h_{0}(X) \leq 4 \delta \log (n+1),
$$

voir la Définition 3.4 et la Définition 3.5 pour les définitions des deux hauteurs dans l'inégalité (9).

Pour comparer la hauteur classique et la hauteur de Philippon d'une hypersurface, il faut comparer la mesure de Mahler et la valeur absolue maximale des coefficients du polynôme qui définit l'hypersurface, où une place infinie $v \in M_{K, \infty}$ est fixée. On utilise la méthode dans [16, §B.7].

Proposition 3.6 Soient $\overline{\mathcal{E}}$ défini dans (7), et $X$ une hypersurface de $\mathbb{P}\left(\mathcal{E}_{K}\right)$ de degré $\delta$. Alors on $a$

$$
-\frac{1}{2} \log ((n+1)(\delta+1)) \leq h(X)-h_{P h}(X) \leq(n+1) \delta \log 2,
$$

où la hauteur de Philippon $h_{P h}(X)$ de X est définie dans la Définition 3.4, et la hauteur classique $h(X)$ de $X$ est définie dans la Définition 3.2. 
Proof On suppose que $X$ est défini par le polynôme

$$
f\left(T_{0}, \ldots, T_{n}\right)=\sum_{\substack{\left(i_{0}, \ldots, i_{n}\right) \in \mathbb{N}^{n+1} \\ i_{0}+\cdots+i_{n}=\delta}} a_{i_{0}, \ldots, i_{n}} T_{0}^{i_{0}} \cdots T_{n}^{i_{n}},
$$

et $d_{i}=\operatorname{deg}_{T_{i}}(f)$ pour tout $i=0, \ldots, n$. D'après [16, Lemma B.7.3.1, Lemma B.7.3.2], soit $v \in M_{K, \infty}$, on a

$$
\begin{aligned}
\prod_{v \in M_{K, \infty}} \frac{M(v(f))}{\sqrt{\left(d_{0}+1\right) \cdots\left(d_{n}+1\right)}} & \leq \prod_{v \in M_{K, \infty}} \max _{\begin{array}{c}
\left(i_{0}, \ldots, i_{n}\right) \in \mathbb{N}^{n+1} \\
i_{0}+\cdots+i_{n}=\delta
\end{array}}\left\{\left|v\left(a_{i_{0}, \ldots, i_{n}}\right)\right|\right\} \\
& \leq \prod_{v \in M_{K, \infty}} 2^{(n+1) \delta} M(v(f)),
\end{aligned}
$$

où la mesure de Mahler $M(v(f))$ est définie dans (2). Si $v \in M_{K, f}$, alors

$$
\max _{\substack{\left(i_{0}, \ldots, i_{n}\right) \in \mathbb{N}^{n+1} \\ i_{0}+\cdots+i_{n}=\delta}}\left\{\left|a_{i_{0}, \ldots, i_{n}}\right|_{v}\right\}=\|f\|_{v} .
$$

Donc par la Définition 3.4 et la Définition 3.2, on obtient le résultat, car $d_{i} \leq \delta$ pour tout $i \in\{0,1, \ldots, n\}$.

On a le résultat suivant en combinant les estimations ci-dessus.

Proposition 3.7 Soient $\overline{\mathcal{E}}$ défini dans (7), X un sous-schéma fermé de dimension pure de $\mathbb{P}\left(\mathcal{E}_{K}\right)$ de dimension $d$ et de degré $\delta$, et $\psi_{X} \in \operatorname{Sym}^{\delta}\left(\Lambda^{d+1} \mathcal{E}_{K}^{\vee}\right)$ l'élément qui définit la variété de Cayley de X (voir la Proposition 2.2 et la Définition 3.3). Alors on a

$$
\begin{aligned}
-\frac{1}{2} \log ((N+1)(\delta+1))-\frac{1}{2} \delta \mathcal{H}_{N} & \leq h\left(\psi_{X}\right)-h \overline{\mathcal{O}(1)}(X) \\
& \leq(N+1) \delta \log 2+4 \delta \log (N+1)-\frac{1}{2} \delta \mathcal{H}_{N},
\end{aligned}
$$

où $h\left(\psi_{X}\right)$ est défini dans la Définition 3.2, $h_{\overline{\mathcal{O}(1)}}(X)$ est défini dans la Définition 3.1, $N=$ $\left(\begin{array}{l}n+1 \\ d+1\end{array}\right)-1$, et $\mathcal{H}_{N}=1+\cdots+\frac{1}{N}$.

Proof Soit $X^{\prime}$ l'hypersurface projective définie par $\psi_{X}$ dans $\mathbb{P}\left(\bigwedge^{d+1} \mathcal{E}_{K}^{\vee}\right)$. On compare la hauteur de Philippon (voir la Définition 3.4) de $X^{\prime}$ et la hauteur classique de $X^{\prime}$. D'après la Proposition 3.6, on a

$$
-\frac{1}{2} \log ((N+1)(\delta+1)) \leq h\left(\psi_{X}\right)-h_{P h}\left(X^{\prime}\right) \leq(N+1) \delta \log 2 .
$$

On compare la 0-hauteur (voir la Définition 3.5) de $X^{\prime}$ et la hauteur de Philippon de $X^{\prime}$. D'après (9), on a

$$
0 \leq h_{P h}\left(X^{\prime}\right)-\widetilde{h}_{0}\left(X^{\prime}\right) \leq 4 \delta \log (N+1) .
$$

Par (8), on a

$$
\widetilde{h}_{0}\left(X^{\prime}\right)=h \overline{\mathcal{O}(1)}(\mathscr{X})-\frac{1}{2} \delta \mathcal{H}_{N} .
$$

On combine (10), (11) et (12), on obtient le résultat. 


\subsection{Hauteur adélique}

Dans cette partie, on introduira une fonction hauteur de la version adélique d'une hypersurface.

\section{Rappel de l'anneau adélique}

D'abord, on rappelle la définition de l'anneau adélique. Étant donnés un corps de nombre $K$ et son anneau des entiers $\mathcal{O}_{K}$, on désgine par

$$
\mathbb{A}_{K}=\left\{\left(a_{v}\right)_{v} \in \prod_{v \in M_{K}} K_{v} \mid a_{v} \in \mathcal{O}_{K, v} \text { sauf pour un nombre fini de } v \in M_{K, f}\right\}
$$

l'anneau adélique de $K$, et par

$$
\mathbb{A}_{\mathcal{O}_{K}}=\left\{\left(a_{v}\right)_{v} \in \mathbb{A}_{K} \mid a_{v} \in \mathcal{O}_{K, v} \text { pour tout } v \in M_{K, f}\right\}
$$

l'anneau adélique des entiers de $K$, voir [18, Chap. VI, §1] pour une introduction autonome de ces notions.

Soit $c \in K$. On désigne par $\Delta(c)$ son image dans $\mathbb{A}_{K}$ par rapport au plongement diagonal $\Delta: K \hookrightarrow \mathbb{A}_{K}$. De plus, soit $a=\left(a_{v}\right)_{v \in M_{K}} \in \mathbb{A}_{K}$, on définit

$$
|a|_{\mathbb{A}_{K}}=\prod_{v \in M_{K}}\left|a_{v}\right|_{v}^{\left[K_{v}: \mathbb{Q}_{v}\right]}
$$

Par la formule de produit (cf. [18, Chap III, (1.3)Proposition]), on a $|\Delta(a)|_{\mathbb{A}_{K}}=1$ pour tout $a \in K^{\times}$.

On a le lemme suivant sur l'existance d'un élément particulier dans $\mathbb{A}_{K}$.

Lemma 3.8 Soit $\mathfrak{a}$ un idéal fractionnaire de $\mathcal{O}_{K}$ avec la décomposition $\mathfrak{p}_{1}^{n_{1}} \cdots \mathfrak{p}_{k}^{n_{k}}$, où $\mathfrak{p}_{1}, \ldots, \mathfrak{p}_{k}$ sont des idéaux primiers de $\mathcal{O}_{K}$ et $n_{1}, \ldots, n_{k} \in \mathbb{Z}$. Alors il existe un élément $a=\left(a_{v}\right)_{v \in M_{K}} \in \mathbb{A}_{K}$, tel que $|a|_{\mathbb{A}_{K}}=1$ et à la place $v \in M_{K, f}$ par rapport à $\mathfrak{p}_{i}, a_{v}$ engendre le même idéal fractionnaire que $\mathfrak{p}_{i}^{n_{i}} \mathcal{O}_{K, \mathfrak{p}_{i}}$ dans $K_{\mathfrak{p}_{i}}$.

Proof Pour chaque $\mathfrak{p}_{i} \in \operatorname{Spm} \mathcal{O}_{K}$, soit $\omega_{i}$ une uniformisante de $\mathcal{O}_{K, \mathfrak{p}_{i}}$, dont l'existance est en raison du fait que $\mathcal{O}_{K, \mathfrak{p}_{i}}$ est un anneau principal. Afin de construire un tel $a=\left(a_{v}\right)_{v \in M_{K}}$, pour une place $v \in M_{K, f}$ par rapport à $\mathfrak{p}_{i}$, on pose $a_{v}=\omega_{i}^{n_{i}}$. Pour les autre places finies $v$, on pose $a_{v}=1$.

Pour toute place $v \in M_{K, \infty}$, on a $K_{v} \cong \mathbb{R}$ ou $\mathbb{C}$. Alors on pose

$$
a_{v}=\left|\prod_{i=1}^{k} \#\left(\mathcal{O}_{K} / \mathfrak{p}_{i}\right)^{n_{i}}\right|^{1 /[K: \mathbb{Q}]}
$$

pour toute place $v \in M_{K, \infty}$. Par un calcul élémentaire, cet élément $\left(a_{v}\right)_{v \in M_{K}}$ satisfait l'assertion.

\section{Hauteur sur I'anneau adélique}

Dans la suite, on considère un polynôme sur l'anneau adélique. Pour introduire une fonction hauteur, on va définir ses parties locales comme suit. 
Definition 3.9 (Partie locale) Soient $\left\{a_{i_{0}, \ldots, i_{n}}\right\}=\left\{\left(a_{i_{0}, \ldots, i_{n}}^{v}\right)_{v \in M_{K}}\right\}$ une famille finie des éléments de $\mathbb{A}_{K}$ avec les indices $\left(i_{0}, \ldots, i_{n}\right) \in \mathbb{N}^{n+1}$, et

$$
F\left(T_{0}, \ldots, T_{n}\right)=\sum_{\left(i_{0}, \ldots, i_{n}\right) \in \mathbb{N}^{n+1}} a_{i_{0}, \ldots, i_{n}} T_{0}^{i_{0}} \cdots T_{n}^{i_{n}} \in \mathbb{A}_{K}\left[T_{0}, \ldots, T_{n}\right]
$$

non-nul. Pour toute place $v \in M_{K}$, on désigne par

$$
F^{(v)}\left(T_{0}, \ldots, T_{n}\right)=\sum_{\left(i_{0}, \ldots, i_{n}\right) \in \mathbb{N}^{n+1}} a_{i_{0}, \ldots, i_{n}}^{v} T_{0}^{i_{0}} \cdots T_{n}^{i_{n}}
$$

la $v$-partie de $F\left(T_{0}, \ldots, T_{n}\right)$, ou par $F^{(\mathfrak{p})}\left(T_{0}, \ldots, T_{n}\right)$ pour le $\mathfrak{p} \in \operatorname{Spm} \mathcal{O}_{K}$ correspondant à une place $v \in M_{K, f}$ qui est appelé la p-partie de $F\left(T_{0}, \ldots, T_{n}\right)$.

Soient $F$ et $F^{(v)}$ les mêmes que dans la Définition 3.9. Pour une place $v \in M_{K}$, on désigne

$$
\|F\|_{v}=\left\|F^{(v)}\right\|_{v}=\max _{\left(i_{0}, \ldots, i_{n}\right) \in \mathbb{N}^{n}}\left\{\left|a_{i_{0}, \ldots, i_{n}}^{v}\right|_{v}\right\},
$$

ou par $\|F\|_{\mathfrak{p}}$ et $\left\|F^{(\mathfrak{p})}\right\|_{\mathfrak{p}}$ pour le $\mathfrak{p} \in \operatorname{Spm} \mathcal{O}_{K}$ correspondant à une place finie. De plus, pour une place infinie $v \in M_{K, \infty}$, on désigne

$$
\|F\|_{2, v}=\left\|F^{(v)}\right\|_{2, v}=\left(\sum_{\left(i_{0}, \ldots, i_{n}\right) \in \mathbb{N}^{n}}\left|a_{i_{0}, \ldots, i_{n}}^{v}\right|_{v}^{2}\right)^{\frac{1}{2}}
$$

Avec les notations au-dessus, on introduit une fonction hauteur comme ci-dessous.

Definition 3.10 (Hauteur adélique) Soit $F$ le même que dans la Définition 3.9. Les deux hauteurs adéliques de $F$ sont définies comme

$$
H_{\mathbb{A}_{K}}(F)=\prod_{v \in M_{K}}\|F\|_{v}^{\left[K_{v}: \mathbb{Q}_{v}\right]}
$$

et

$$
H_{\mathbb{A}_{K}, 2}(F)=\prod_{v \in M_{K, f}}\|F\|_{v}^{\left[K_{v}: \mathbb{Q}_{v}\right]} \cdot \prod_{v \in M_{K, \infty}}\|F\|_{2, v}^{\left[K_{v}: \mathbb{Q}_{v}\right]} .
$$

De plus, on définit les hauteurs logarithmiquement adéliques comme

$$
h(F)=\frac{1}{[K: \mathbb{Q}]} \log H_{\mathbb{A}_{K}}(F) \text { et } h_{2}(F)=\frac{1}{[K: \mathbb{Q}]} \log H_{\mathbb{A}_{K}, 2}(F)
$$

respectivement.

En suite, on introduit la notion de la hauteur infinie.

Definition 3.11 [Hauteur infinie adélique] Soit $F$ le même que celui dans la Définition 3.9. Les deux hauteurs infinies adéliques de $F$ sont définies comme

$$
H_{\infty, \mathbb{A}_{K}}(F)=\prod_{v \in M_{K, \infty}}\|F\|_{v}^{\left[K_{v}: \mathbb{Q}_{v}\right]} \text { et } H_{\infty, \mathbb{A}_{K}, 2}(F)=\prod_{v \in M_{K, \infty}}\|F\|_{2, v}^{\left[K_{v}: \mathbb{Q}_{v}\right]} .
$$

De plus, on définit les hauteurs infinies logarithmiquement adéliques comme

$$
h_{\infty}(F)=\frac{1}{[K: \mathbb{Q}]} \log H_{\infty, \mathbb{A}_{K}}(F) \text { et } h_{\infty, 2}(F)=\frac{1}{[K: \mathbb{Q}]} \log H_{\infty, \mathbb{A}_{K}, 2}(F)
$$

respectivement. 
Remarque 3.12 Par un calcul élémentaire, la hauteur adélique est invariante sous la multiplication d'un élément $a \in \mathbb{A}_{K}$ avec $|a|_{\mathbb{A}_{K}}=1$, et est bien sûr invariante sous la multiplication d'un élément d'un élément dans $K^{\times}$considéré comme un élément dans $\mathbb{A}_{K}$ par rapport au plongement diagonal $K \hookrightarrow \mathbb{A}_{K}$.

Soient $f \in K\left[T_{0}, \ldots, T_{n}\right]$, et $F$ l'image canonique de $f$ dans $\mathbb{A}_{K}\left[T_{0}, \ldots, T_{n}\right]$ par rapport au plongement diagonal $K \hookrightarrow \mathbb{A}_{K}$. Alors on a

$$
H_{K}(f)=H_{\mathbb{A}_{K}}(F) \text { et } H_{K, 2}(f)=H_{\mathbb{A}_{K}, 2}(F),
$$

où $H_{K}(f)$ est défini dans la Définition 3.2.

Soit

$$
F\left(T_{0}, \ldots, T_{n}\right)=\sum_{\substack{\left(i_{0}, \ldots, i_{n}\right) \in \mathbb{N}^{n+1} \\ i_{0}+\cdots+i_{n}=\delta}} a_{i_{0}, \ldots, i_{n}} T_{0}^{i_{0}} \cdots T_{n}^{i_{n}} \in \mathbb{A}_{K}\left[T_{0}, \ldots, T_{n}\right] .
$$

Avec toutes les notations dans la Définition 3.10, pour tout $v \in M_{K, \infty}$, on a

$$
\begin{aligned}
\max _{\substack{\left(i_{0}, \ldots, i_{n}\right) \in \mathbb{N}^{n+1} \\
i_{0}+\cdots+i_{n}=\delta}}\left\{\left|a_{i_{0}, \ldots, i_{n}}^{v}\right|_{v}\right\} & \leq\left(\sum_{\substack{\left(i_{0}, \ldots, i_{n}\right) \in \mathbb{N}^{n+1} \\
i_{0}+\cdots+i_{n}=\delta}}\left|a_{i_{0}, \ldots, i_{n}}^{v}\right|_{v}^{2}\right)^{\frac{1}{2}} \\
& \leq\left(\begin{array}{c}
n+\delta \\
n
\end{array}\right)^{\frac{1}{2}} \max _{\substack{\left.i_{0}, \ldots, i_{n}\right) \in \mathbb{N}^{n+1} \\
i_{0}+\cdots+i_{n}=\delta}}\left\{\left|a_{i_{0}, \ldots, i_{n}}^{v}\right|_{v}\right\}
\end{aligned}
$$

par la définition directement. Donc on a le résultat suivant immédiatement.

Lemma 3.13 Avec toutes les notations dans la Définition 3.10 et la Définition 3.11. Soit $F \in \mathbb{A}_{K}\left[T_{0}, \ldots, T_{n}\right]$. Alors on a

$$
0 \leq h_{2}(F)-h(F) \leq \frac{1}{2} \log \left(\begin{array}{c}
n+\delta \\
n
\end{array}\right)
$$

et

$$
0 \leq h_{\infty, 2}(F)-h_{\infty}(F) \leq \frac{1}{2} \log \left(\begin{array}{c}
n+\delta \\
n
\end{array}\right)
$$

\section{Une estimation de l'annulation de résultant par réductions}

Dans cette section, pour un polynôme sur un corps de nombres $K$, on donnera une majoration de l'annulation d'un de ses résultants par réductions. Cette majoration dépend la hauteur, le degré et le nombre des variables de ce polynôme.

\subsection{Résultant sur un anneau}

Soient $A$ un anneau, et

$$
f\left(T_{0}, \ldots, T_{n}\right)=\sum_{\substack{\left(i_{0}, \ldots, i_{n}\right) \in \mathbb{N}^{n+1} \\ i_{0}+\cdots+i_{n}=\delta}} a_{i_{0}, \ldots, i_{n}} T_{0}^{i_{0}} \cdots T_{n}^{i_{n}} \in A\left[T_{0}, \ldots, T_{n}\right]
$$


un polynôme homogène de degré $\delta$. On désigne par $d_{i}=\operatorname{deg}_{T_{i}}(f)$ pour $i=0, \ldots, n$, et on suppose $d_{n}>0$ sans perte de généralité. On écrit $f\left(T_{0}, \ldots, T_{n}\right)$ sous la forme de

$$
f\left(T_{0}, \ldots, T_{n}\right)=s_{d_{n}}\left(T_{0}, \ldots, T_{n-1}\right) T_{n}^{d_{n}}+\cdots+s_{0}\left(T_{0}, \ldots, T_{n-1}\right),
$$

où l'on a $s_{d_{n}}\left(T_{0}, \ldots, T_{n-1}\right) \neq 0$.

Par (15), on considère $f\left(T_{0}, \ldots, T_{n}\right)$ comme un polynôme à coefficients dans l'anneau $A\left[T_{0}, \ldots, T_{n-1}\right]$ de degré $d_{n}$, alors $\frac{\partial f}{\partial T_{n}}$ est de degré $d_{n}-1$ à coefficients dans $A\left[T_{0}, \ldots, T_{n-1}\right]$ si $A$ est de caractérisque nulle. Par le sens ci-dessus, le résultant de $f\left(T_{0}, \ldots, T_{n}\right)$ et $\frac{\partial f}{\partial T_{n}}$ est

$$
\begin{aligned}
& \operatorname{Res}_{d_{n}}\left(f, \frac{\partial f}{\partial T_{n}}\right)
\end{aligned}
$$

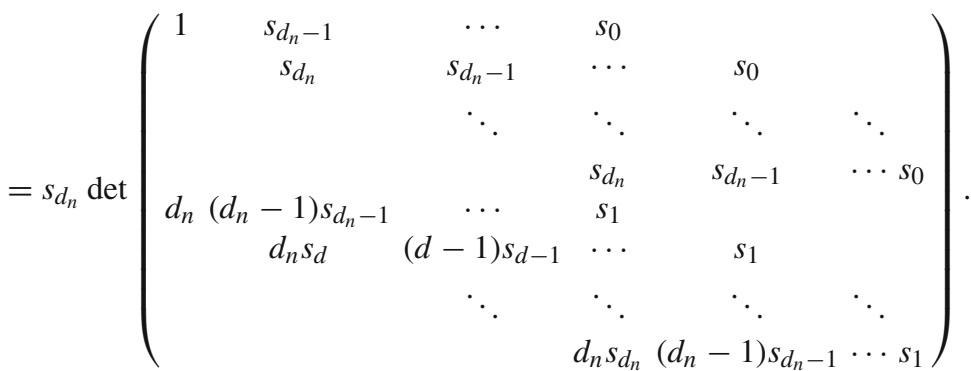

On désigne

$$
\begin{aligned}
& \operatorname{Res}_{d_{n}}^{\prime}\left(f, \frac{\partial f}{\partial T_{n}}\right) \\
& =\operatorname{det}\left(\begin{array}{cccccc}
1 & s_{d_{n}-1} & \cdots & s_{0} & & \\
& s_{d_{n}} & s_{d_{n}-1} & \cdots & s_{0} & \\
& & \ddots & \ddots & \ddots & \ddots \\
& & & s_{d_{n}} & s_{d_{n}-1} & \cdots s_{0} \\
d_{n}\left(d_{n}-1\right) s_{d_{n}-1} & \cdots & s_{1} & & \\
& d_{n} s_{d_{n}} & \left(d_{n}-1\right) s_{d_{n}-1} & \cdots & s_{1} & \\
& & \ddots & \ddots & \ddots & \ddots \\
& & & d_{n} s_{d_{n}}\left(d_{n}-1\right) s_{d_{n}-1} \cdots s_{1}
\end{array}\right)
\end{aligned}
$$

pour simplifier. Par la construction ci-dessus, on a $\operatorname{Res}_{d_{n}}^{\prime}\left(f, \frac{\partial f}{\partial T_{n}}\right) \in A\left[T_{0}, \ldots, T_{n-1}\right]$.

\subsection{Polynôme adéliquement primitif}

Maintenant on travaille sur un corp de nombre $K$ et son anneau des entiers $\mathcal{O}_{K}$. Soit $f \in$ $K\left[T_{0}, \ldots, T_{n}\right]$ un polynôme homogène non-nul de degré $\delta$. On maintient toutes les notations même que celles dans $\S 4.1$ lorsque $A$ est $K, \mathbb{A}_{K}$ ou $\mathbb{A}_{\mathcal{O}_{K}}$.

On considère $f\left(T_{0}, \ldots, T_{n}\right)$ comme un polynôme à coefficients dans $\mathbb{A}_{K}$ par rapport au plongement diagonal $\Delta: K \hookrightarrow \mathbb{A}_{K}$. Par le Lemme 3.8, il existe un élément $c=\left(c_{v}\right)_{v \in M_{K}} \in$ $\mathbb{A}_{K}$ avec $|c|_{\mathbb{A}_{K}}=1$ (voir (13) pour la définition de $|\cdot|_{\mathbb{A}_{K}}$ ), tel que pour toute place $v \in M_{K, f}$, on ait

$$
\max _{\substack{\left(i_{0}, \ldots, i_{n}\right) \in \mathbb{N}^{n+1} \\ i_{0}+\cdots+i_{n}=\delta}}\left\{\left|c \Delta\left(a_{i_{0}, \ldots, i_{n}}\right)\right|_{v}\right\}=1 .
$$


On désigne par $b_{i_{0}, \ldots, i_{n}}=\left(b_{i_{0}, \ldots, i_{n}}^{v}\right)_{v}=c \Delta\left(a_{i_{0}, \ldots, i_{n}}\right)$ pour simplifier, et on dit que

$$
F\left(T_{0}, \ldots, T_{n}\right)=\sum_{\substack{\left(i_{0}, \ldots, i_{n}\right) \in \mathbb{N}^{n+1} \\ i_{0}+\cdots+i_{n}=\delta}} b_{i_{0}, \ldots, i_{n}} T_{0}^{i_{0}} \cdots T_{n}^{i_{n}}
$$

est le polynôme adéliquement primitif de $f\left(T_{0}, \ldots, T_{n}\right)$. En effet, on a $F\left(T_{0}, \ldots, T_{n}\right) \in$ $\mathbb{A}_{\mathcal{O}_{K}}\left[T_{0}, \ldots, T_{n}\right]$, et sa composante en toute place finie est un polynôme primitif au sens habituel. Dans ce cas-là, $F$ est de degré $d_{n}$ en la variable $T_{n}$.

Pour un polynôme adéliquement primitif $F$, on a

$$
h(F)=h_{\infty}(F), \text { et } h_{2}(F)=h_{\infty, 2}(F)
$$

où $h(F)$ et $h_{2}(F)$ sont définis dans la Définition 3.10, et $h_{\infty}(F)$ et $h_{\infty, 2}(f)$ sont définis dans la Définition 3.11.

\subsection{L'estimation d'une hauteur du résultant}

Soit

$$
G\left(T_{0}, \ldots, T_{n-1}\right)=\sum_{\left(i_{0}, \ldots, i_{n-1}\right) \in \mathbb{N}^{n}} b_{i_{0}, \ldots, i_{n-1}} T_{0}^{i_{0}} \cdots T_{n-1}^{i_{n-1}}
$$

un polynôme non-nul à coefficients dans $\mathbb{A}_{K}$, où l'on désigne $b_{i_{0}, \ldots, i_{n-1}}=\left(b_{i_{0}, \ldots, i_{n-1}}^{v}\right)_{v \in M_{K}}$. Pour tout $\mathfrak{p} \in \operatorname{Spm} \mathcal{O}_{K}$ correspondant à la place $v \in M_{K, f}$, on définit sa norme donnée par le modèle comme

$$
\|G\|_{\mathfrak{p}}=\left\|G^{(\mathfrak{p})}\right\|_{\mathfrak{p}}=\max _{\left(i_{0}, \ldots, i_{n-1}\right) \in \mathbb{N}^{n}}\left\{\left|b_{i_{0}, \ldots, i_{n-1}}^{v}\right|_{v}\right\},
$$

où la p-partie $G^{(\mathfrak{p})}$ est définie dans la Définition 3.9. Si $G \in \mathbb{A}_{\mathcal{O}_{K}}\left[T_{0}, \ldots, T_{n-1}\right]$, on désigne

$$
\mathcal{P}(G)=\left\{\mathfrak{p} \in \operatorname{Spm} \mathcal{O}_{K} \mid G^{(\mathfrak{p})} \bmod \mathfrak{p}\left[T_{0}, \ldots, T_{n-1}\right]=0\right\} .
$$

Pour une place infinie $v \in M_{K, \infty}$, on désigne

$$
\|G\|_{v}=\max _{\left(i_{0}, \ldots, i_{n-1}\right) \in \mathbb{N}^{n}}\left\{\left|b_{i_{0}, i_{1}, \ldots, i_{n-1}}^{v}\right|_{v}\right\} \text { et }\|G\|_{2, v}=\left(\sum_{\left(i_{0}, \ldots, i_{n-1}\right) \in \mathbb{N}^{n}}\left|b_{i_{0}, i_{1}, \ldots, i_{n-1}}^{v}\right|_{v}^{2}\right)^{\frac{1}{2}}
$$

qui sont les mêmes que ceux dans $\S 3.4$.

Soient $G_{1}, \ldots, G_{m} \in \mathbb{A}_{K}\left[T_{0}, \ldots, T_{n-1}\right]$. Pour toute place $v \in M_{K, \infty}$, on définit

$$
\left\|\left(G_{1}, \ldots, G_{m}\right)\right\|_{2, v}=\sqrt{\left\|G_{1}\right\|_{2, v}^{2}+\cdots+\left\|G_{m}\right\|_{2, v}^{2}} .
$$

Pour le polynôme $F\left(T_{0}, \ldots, T_{n}\right)$ défini dans (18), on définit $\operatorname{Res}_{d_{n}}^{\prime}\left(F, \frac{\partial F}{\partial T_{n}}\right) \in$ $\mathbb{A}_{\mathcal{O}_{K}}\left[T_{0}, \ldots, T_{n-1}\right]$ comme (17). Afin d'estimer la taille de l'ensemble $\mathcal{P}\left(\operatorname{Res}_{d_{n}}^{\prime}\left(F, \frac{\partial F}{\partial T_{n}}\right)\right)$ défini dans (20), on a le résultat suivant.

Proposition 4.1 Avec toutes les notations et constructions au-dessus. Soit $F\left(T_{0}, \ldots, T_{n}\right)$ déterminé dans (18) sur $\mathbb{A}_{\mathcal{O}_{K}}$ à partir de $f\left(T_{0}, \ldots, T_{n}\right)$ dans (15). Pour tout $\mathfrak{p} \in \operatorname{Spm} \mathcal{O}_{K}$, 
on désigne $N(\mathfrak{p})=\#\left(\mathcal{O}_{K} / \mathfrak{p}\right)$. Alors on a

$$
\begin{aligned}
& \frac{1}{[K: \mathbb{Q}]} \sum_{\mathfrak{p} \in \mathcal{P}\left(\operatorname{Res}_{d_{n}}^{\prime}\left(F, \frac{\partial F}{\partial T_{n}}\right)\right)} \log N(\mathfrak{p}) \\
& \leq\left(2 d_{n}-2\right) h(f)+\left(d_{n}-1\right) \log \left(\begin{array}{c}
n+\delta \\
n
\end{array}\right)+\log \left(2 d_{n}^{d_{n}}-d_{n}^{d_{n}-1}\right) .
\end{aligned}
$$

où $h(f)$ est défini dans la Définition 3.2.

Proof Si $d_{n}=\max \left\{d_{0}, \ldots, d_{n}\right\}=1$, on a $\operatorname{Res}_{d_{n}}^{\prime}\left(F, \frac{\partial F}{\partial T_{n}}\right)=1$ par définition directement. Donc l'ensemble $\mathcal{P}\left(\operatorname{Res}_{d_{n}}^{\prime}\left(F, \frac{\partial F}{\partial T_{n}}\right)\right)$ est vide, qui satisfait l'inégalité dans l'énoncé.

Dans le reste de la démonstration, on suppose $d_{n} \geq 2$. Comme $\operatorname{Res}_{d_{n}}^{\prime}\left(F, \frac{\partial F}{\partial T_{n}}\right) \in$ $\mathbb{A}_{\mathcal{O}_{K}}\left[T_{0}, \ldots, T_{n-1}\right]$, alors pour tout $\mathfrak{p} \in \operatorname{Spm} \mathcal{O}_{K}$, on a

$$
\left\|\operatorname{Res}_{d_{n}}^{\prime}\left(F, \frac{\partial F}{\partial T_{n}}\right)^{(\mathfrak{p})}\right\|_{\mathfrak{p}} \leq 1 .
$$

Donc on a

$$
\begin{aligned}
& \frac{1}{[K: \mathbb{Q}]} \sum_{\mathfrak{p} \in \mathcal{P}\left(\operatorname{Res}_{d_{n}}^{\prime}\left(F, \frac{\partial F}{\partial T_{n}}\right)\right)} \log N(\mathfrak{p}) \\
& \leq-\sum_{\mathfrak{p} \in \mathcal{P}\left(\operatorname{Res}_{d_{n}}^{\prime}\left(F, \frac{\partial F}{\partial T_{n}}\right)\right)} \frac{\left[K_{\mathfrak{p}}: \mathbb{Q} \mathfrak{p}\right]}{[K: \mathbb{Q}]} \log \left\|\operatorname{Res}_{d_{n}}^{\prime}\left(F, \frac{\partial F}{\partial T_{n}}\right)^{(\mathfrak{p})}\right\|_{\mathfrak{p}} .
\end{aligned}
$$

De plus, l'inégalité

$$
\left\|\operatorname{Res}_{d_{n}}^{\prime}\left(F, \frac{\partial F}{\partial T_{n}}\right)^{(\mathfrak{p})}\right\|_{\mathfrak{p}}<1
$$

est vérifiée si et seulement si $\mathfrak{p} \in \mathcal{P}\left(\operatorname{Res}_{d_{n}}^{\prime}\left(F, \frac{\partial F}{\partial T_{n}}\right)\right)$. Alors on obtient

$$
\begin{aligned}
& -\sum_{\mathfrak{p} \in \mathcal{P}\left(\operatorname{Res}_{d_{n}}^{\prime}\left(F, \frac{\partial F}{\partial T_{n}}\right)\right)} \frac{\left[K_{\mathfrak{p}}: \mathbb{Q}_{\mathfrak{p}}\right]}{[K: \mathbb{Q}]} \log \left\|\operatorname{Res}_{d_{n}}^{\prime}\left(F, \frac{\partial F}{\partial T_{n}}\right)^{(\mathfrak{p})}\right\|_{\mathfrak{p}} \\
& =-\sum_{\mathfrak{p} \in \operatorname{Spm}_{\mathcal{O}_{K}}} \frac{\left[K_{\mathfrak{p}}: \mathbb{Q}_{\mathfrak{p}}\right]}{[K: \mathbb{Q}]} \log \left\|\operatorname{Res}_{d_{n}}^{\prime}\left(F, \frac{\partial F}{\partial T_{n}}\right)^{(\mathfrak{p})}\right\|_{\mathfrak{p}} \\
& =-h\left(\operatorname{Res}_{d_{n}}^{\prime}\left(F, \frac{\partial F}{\partial T_{n}}\right)\right)+\sum_{v \in M_{K, \infty}} \frac{\left[K_{v}: \mathbb{Q}_{v}\right]}{[K: \mathbb{Q}]} \log \left\|\operatorname{Res}_{d_{n}}^{\prime}\left(F, \frac{\partial F}{\partial T_{n}}\right)^{(v)}\right\|_{v} .
\end{aligned}
$$

En effet, le polynôme $\operatorname{Res}_{d_{n}}^{\prime}\left(F, \frac{\partial F}{\partial T_{n}}\right) \in \mathbb{A}_{K}\left[T_{0}, \ldots, T_{n-1}\right]$ est obtenu par la multiplication d'un élément $c \in \mathbb{A}_{K}$ à un polynôme dans $K\left[T_{0}, \ldots, T_{n-1}\right]$ satisfaisant $|c|_{\mathbb{A}_{K}}=1$. Alors d'après la Remarque 3.12 et la Définition 3.2, on a $h\left(\operatorname{Res}_{d_{n}}^{\prime}\left(F, \frac{\partial F}{\partial T_{n}}\right)\right) \geq 0$. On le combine 
avec (14), et on a

$$
\begin{aligned}
& -\sum_{\mathfrak{p} \in \mathcal{P}\left(\operatorname{Res}_{d_{n}}^{\prime}\left(F, \frac{\partial F}{\partial T_{n}}\right)\right)} \frac{\left[K_{\mathfrak{p}}: \mathbb{Q}_{\mathfrak{p}}\right]}{[K: \mathbb{Q}]} \log \left\|\operatorname{Res}_{d_{n}}^{\prime}\left(F, \frac{\partial F}{\partial T_{n}}\right)^{(\mathfrak{p})}\right\|_{\mathfrak{p}} \\
& \leq \sum_{v \in M_{K, \infty}} \frac{\left[K_{v}: \mathbb{Q}_{v}\right]}{[K: \mathbb{Q}]} \log \left\|\operatorname{Res}_{d_{n}}^{\prime}\left(F, \frac{\partial F}{\partial T_{n}}\right)^{(v)}\right\|_{2, v} .
\end{aligned}
$$

d'après l'égalité ci-dessus.

Afin d'estimer $\left\|\operatorname{Res}_{d_{n}}^{\prime}\left(F, \frac{\partial F}{\partial T_{n}}\right)^{(v)}\right\|_{2, v}$ pour une place $v \in M_{K, \infty}$, on renvoie que c'est la $v$-partie de $\operatorname{Res}_{d_{n}}^{\prime}\left(F, \frac{\partial F}{\partial T_{n}}\right)$ définie dans la matrice de (17) sur l'anneau $\mathbb{A}_{\mathcal{O}_{K}}$. Soit $w_{i}^{v}$ le $i$-ième vecteur de ligne dans la $v$-partie de la matrice supprimant la première colonne dans (17), où $i=1, \ldots, 2 d_{n}-1$. Alors on écrit la matrice sous la forme de

$$
\operatorname{Res}_{d_{n}}^{\prime}\left(F, \frac{\partial F}{\partial T_{n}}\right)^{(v)}=\operatorname{det}\left(\begin{array}{cc}
1 & w_{1}^{v} \\
& \vdots \\
& w_{d_{n}-1}^{v} \\
d_{n} & w_{d_{n}}^{v} \\
& \vdots \\
& w_{2 d_{n}-1}^{v}
\end{array}\right) .
$$

D'abord on développe la matrice ci-dessus par rapport à la permière colonne, qui a deux éléments non-nuls seulement. Soient $M_{v}$ et $N_{v}$ les cofacteurs de 1 et $d_{n}$ respectivement dans cette matrice. Alors on a

$$
M_{v}=\left(\begin{array}{c}
w_{2}^{v} \\
\vdots \\
w_{2 d_{n}-1}^{v}
\end{array}\right) \text { et } N_{v}=\left(\begin{array}{c}
w_{1}^{v} \\
\vdots \\
w_{d_{n}-1}^{v} \\
w_{d_{n}+1}^{v} \\
\vdots \\
w_{2 d_{n}-1}^{v}
\end{array}\right) .
$$

Par définition, on a

$$
\left\|\operatorname{Res}_{d_{n}}^{\prime}\left(F, \frac{\partial F}{\partial T_{n}}\right)^{(v)}\right\|_{2, v} \leq\left\|\operatorname{det}\left(M_{v}\right)\right\|_{2, v}+d_{n}\left\|\operatorname{det}\left(N_{v}\right)\right\|_{2, v} .
$$

En raison du fait que la norme $\|\cdot\|_{2, v}$ est hermitienne sur $K_{v}\left[T_{0}, \ldots, T_{n}\right]$, d'après $[2$, Corollaire 1, §3.5 Chap. V], on a

$$
\left\|\operatorname{det}\left(M_{v}\right)\right\|_{2, v} \leq \prod_{i=2}^{2 d_{n}-1}\left\|w_{i}^{v}\right\|_{2, v} \text { et }\left\|\operatorname{det}\left(N_{v}\right)\right\|_{2, v} \leq \frac{1}{\left\|w_{d_{n}}^{v}\right\|_{2, v}} \prod_{i=1}^{2 d_{n}-1}\left\|w_{i}^{v}\right\|_{2, v}
$$

où chaque $\left\|w_{i}^{v}\right\|_{2, v}$ est défini dans (21) pour $i=1, \ldots, 2 d_{n}-1$.

Par définition, on a $\left\|w_{i}^{v}\right\|_{2, v} \leq\|F\|_{2, v}$ pour les $i=1, \ldots, d_{n}-1,\left\|w_{d_{n}}^{v}\right\|_{2, v} \leq\left(d_{n}-\right.$ 1) $\|F\|_{2, v}$, et $\left\|w_{i}^{v}\right\|_{2, v} \leq d_{n}\|F\|_{2, v}$ pour les $i=d_{n}+1, \ldots, 2 d_{n}-1$. Alors on obtient

$$
\left\|\operatorname{det}\left(M_{v}\right)\right\|_{2, v} \leq\left(d_{n}-1\right) d_{n}^{d_{n}-1}\|F\|_{2, v}^{2 d_{n}-2} \text { et } d_{n}\left\|\operatorname{det}\left(N_{v}\right)\right\|_{2, v} \leq d_{n}^{d_{n}}\|F\|_{2, v}^{2 d_{n}-2} .
$$


On combine les estimations ci-dessus, et on obtient

$$
\begin{aligned}
& \sum_{v \in M_{K, \infty}} \frac{\left[K_{v}: \mathbb{Q}_{v}\right]}{[K: \mathbb{Q}]} \log \left\|\operatorname{Res}_{d_{n}}^{\prime}\left(F, \frac{\partial F}{\partial T_{n}}\right)^{(v)}\right\|_{2, v} \\
& \leq\left(2 d_{n}-2\right) \sum_{v \in M_{K, \infty}} \frac{\left[K_{v}: \mathbb{Q}_{v}\right]}{[K: \mathbb{Q}]} \log \|F\|_{2, v}+\log \left(2 d_{n}^{d_{n}}-d_{n}^{d_{n}-1}\right) .
\end{aligned}
$$

Comme $F$ est le polynôme adéliquement primitif de $f$, on a

$$
\sum_{v \in M_{K, \infty}} \frac{\left[K_{v}: \mathbb{Q}_{v}\right]}{[K: \mathbb{Q}]} \log \|F\|_{2, v}=h_{2}(F)
$$

par (19). D'après la Remarque 3.12 et la Proposition 3.13, on a

$$
h_{2}(F) \leq h(F)+\frac{1}{2} \log \left(\begin{array}{c}
n+\delta \\
n
\end{array}\right)=h(f)+\frac{1}{2} \log \left(\begin{array}{c}
n+\delta \\
n
\end{array}\right),
$$

où et $h(f)$ est défini dans la Définition 3.2. Donc on obtient le résultat.

\section{Contrôle des fibres non réduites d'une hypersurface projective}

Soient $\overline{\mathcal{E}}$ un fibré vectoriel hermitien de rang $n+1 \operatorname{sur} \operatorname{Spec} \mathcal{O}_{K}, X$ un sous-schéma fermé de $\mathbb{P}\left(\mathcal{E}_{K}\right)$, et $\mathscr{X}$ l'adhérence schématique de $X$ dans $\mathbb{P}(\mathcal{E})$. Par [13, Théorème (9.7.7)], si $X$ est réduit, alors il n'a y qu'un nombre fini d'ideaux maximaux $\mathfrak{p} \in \operatorname{Spm} \mathcal{O}_{K}$ telles que le fibre $\mathscr{X}_{\mathbb{F}_{\mathfrak{p}}}=\mathscr{X} \times{ }_{\operatorname{Spec}} \mathcal{O}_{K} \operatorname{Spec} \mathbb{F}_{\mathfrak{p}}$ ne soit pas réduite, où $\mathbb{F}_{\mathfrak{p}}$ est le corps résiduel de $\mathcal{O}_{K}$ en $\mathfrak{p}$.

Dans cette section, on donnera une description numérique des réduitions non réduites lorsque $X$ est une hypersurface dans $\mathbb{P}\left(\mathcal{E}_{K}\right)$. Plus précisement, on donnera une majoration du produit des normes des idéaux maximaux $\mathfrak{p} \in \operatorname{Spm} \mathcal{O}_{K}$ tels que $\mathscr{X}_{\mathbb{F}_{\mathfrak{p}}}=\mathscr{X} \times \operatorname{Spec} \mathcal{O}_{K} \operatorname{Spec} \mathbb{F}_{\mathfrak{p}}$ ne soit pas réduit.

\subsection{Résultats préliminaires}

Pour le critère du réduisant d'hypersurface et le choix du plongement dans $\mathbb{P}\left(\mathcal{E}_{K}\right)$, il faut des résultats auxiliaires suivants.

\section{Critère du réduisant d'une hypersurface}

Pour introduire une méthode de critère de réduisant d'une hypersurface projective, d'abord on référence le résultat suivant, qui est une critère du réduisant de l'hypersurface affine.

Lemma 5.1 ([17], Exercise 2.4.1) Soient $k$ un corps, et $P \in k\left[T_{1}, \ldots, T_{n}\right]$ un polynôme nonnul. Alors le schéma Spec $\left(k\left[T_{1}, \ldots, T_{n}\right] /(P)\right)$ est réduit (resp. irréductible; resp. intègre) si et seulement si $P$ n'a pas de facteur carré(resp. est une puissance d'un polynôme irréductible, resp. est irréductible).

Remarque 5.2 D'après le Lemme 5.1, soient $k$ un corps, et $P \in k\left[T_{0}, \ldots, T_{n}\right]$ un polynôme homogène non-nul. Alors $X=\operatorname{Proj}\left(k\left[T_{0}, \ldots, T_{n}\right] /(P)\right)$ est réduit si et seulement si $P$ n'a pas de facteur carré. 


\section{Changement de coordonnée}

Soit $\mathscr{X}$ un $\mathcal{O}_{K}$-schéma réduit, on définit l'ensemble

$$
\mathcal{Q}(\mathscr{X})=\left\{\mathfrak{p} \in \operatorname{Spm} \mathcal{O}_{K} \mid \mathscr{X} \times{ }_{\operatorname{Spec}} \mathcal{O}_{K} \operatorname{Spec} \mathbb{F}_{\mathfrak{p}} \text { ne soit pas réduite }\right\} .
$$

Afin d'appliquer la méthode de résultant pour l'estimation des fibres non réduites d'un schéma arithmétique, il faut choisir une coordonnée particulière. Les deux lemmes suivants sont utiles pour ce but.

Le lemme premier est déduit d'après [14, Proposition(4.4.5), Chap. I] directement.

Lemma 5.3 Soient $\mathcal{E}$ un fibré vectoriel hermitien sur $\operatorname{Spec} \mathcal{O}_{K}, \mathscr{X}$ un sous-schéma fermé réduit de $\mathbb{P}(\mathcal{E})$, et $\sigma \in$ Aut $_{\mathcal{O}_{K}}(\mathbb{P}(\mathcal{E}))$. Alors on a $\mathcal{Q}(\mathscr{X})=\mathcal{Q}(\sigma(\mathscr{X}))$, où $\mathcal{Q}(\cdot)$ est défini dans (22).

Le lemme suivant est une analogie de [9, Lemma 2.1, Lemma 2.2].

Lemma 5.4 Soit $F\left(T_{0}, \ldots, T_{n}\right)$ le polynôme homogène adéliquement primitif determiné à (18). Alors il existe un $\left(a_{0}, \ldots, a_{n-1}\right) \in \mathbb{Z}^{n}$ avec $\max _{0 \leq i \leq n-1}\left\{\left|a_{i}\right|\right\} \leq \frac{\delta+1}{2}$ qui induit un $\sigma \in$ Aut $_{\mathcal{O}_{K}}(\mathbb{P}(\mathcal{E}))$, tel que le coefficient de $T_{n}^{\delta}$ dans $F\left(T_{0}+a_{0} T_{n}, \ldots, T_{n-1}+a_{n-1} T_{n-1}, T_{n}\right) n e$ soit pas zéro, où l'on prolonge $\mathbb{Z}$ dans $\mathbb{A}_{\mathcal{O}_{K}}$ canoniquement. De plus, on a

$$
h(\sigma(\mathscr{X})) \leq h(X)+\log \left(\left(\begin{array}{c}
n+\delta \\
\delta
\end{array}\right)\left(\begin{array}{c}
\delta \\
{\left[\frac{\delta+1}{2}\right]}
\end{array}\right)\left(\frac{\delta+1}{2}\right)^{\delta}\right) .
$$

Proof En effet, le coefficient de $T_{0}^{s_{0}} \cdots T_{n}^{s_{n}}$ dans le polynôme

$$
\begin{aligned}
F & \left(T_{0}+a_{0} T_{n}, \ldots, T_{n-1}+a_{n-1} T_{n-1}, T_{n}\right) \\
& =\sum_{\substack{\left(j_{0}, \ldots, j_{n}\right) \in \mathbb{N}^{n+1} \\
j_{0}+\cdots+j_{n}=\delta}} b_{j_{0}, \ldots, j_{n}}\left(T_{0}+a_{0} T_{n}\right)^{j_{0}} \cdots\left(T_{n-1}+a_{n-1} T_{n}\right)^{j_{n-1}} T_{n}^{j_{n}}
\end{aligned}
$$

est

$$
\sum_{\substack{\left(j_{0}, \ldots, j_{n}\right) \in \mathbb{N}^{n+1} \\
j_{0}+\cdots+j_{n}=\delta}} b_{j_{0}, \ldots, j_{n}}\left(\begin{array}{c}
j_{0} \\
s_{0}
\end{array}\right) \cdots\left(\begin{array}{c}
j_{n-1} \\
s_{n-1}
\end{array}\right) a_{0}^{j_{0}-s_{0}} \cdots a_{n-1}^{j_{n-1}-s_{n-1}}
$$

par un calcul élémentaire. En particulier, le coefficient de $T_{n}^{\delta}$ dans $F\left(T_{0}+a_{0} T_{n}, \ldots, T_{n-1}+\right.$ $a_{n-1} T_{n-1}, T_{n}$ ) est

$$
\sum_{\substack{\left(i_{0}, \ldots, i_{n}\right) \in \mathbb{N}^{n+1} \\ i_{0}+\cdots+i_{n}=\delta}} b_{i_{0}, \ldots, i_{n}} a_{0}^{i_{0}} \cdots a_{n-1}^{i_{n-1}},
$$

qui est un polynôme non-nul de degré au plus $\delta$ de variables $a_{0}, \ldots, a_{n-1}$. De plus, l'ensemble $\left\{-\left[\frac{\delta+1}{2}\right], \ldots,-1,0,1, \ldots,\left[\frac{\delta+1}{2}\right]\right\}^{n}$ est de cardinal plus grand que ou égal à $(\delta+1)^{n}$. Par $[3$, Lemma 1, la page 261], il existe un $\left(a_{0}, \ldots, a_{n-1}\right) \in\left\{-\left[\frac{\delta+1}{2}\right], \ldots,-1,0,1, \ldots,\left[\frac{\delta+1}{2}\right]\right\}^{n}$ qui satisfait le besoin.

Pour l'estimation de la hauteur, $F\left(T_{0}, \ldots, T_{n}\right)$ et $F\left(T_{0}+a_{0} T_{n}, \ldots, T_{n-1}+a_{n-1} T_{n-1}, T_{n}\right)$ sont adéliquement primitifs. De plus, il y a au plus $\left(\begin{array}{c}n+\delta \\ \delta\end{array}\right)$ termes dans la somme (23). En suite, une majoration de $\left(\begin{array}{c}j_{0} \\ s_{0}\end{array}\right) \cdots\left(\begin{array}{c}j_{n-1} \\ s_{n-1}\end{array}\right)$ dans (23) est $\left(\left[\begin{array}{c}\delta \\ \frac{\delta+1}{2}\end{array}\right]\right.$, et une majoration de $a_{0}^{j_{0}-s_{0}} \cdots a_{n-1}^{j_{n-1}-s_{n-1}}$ dans $(23)$ est $\left(\frac{\delta+1}{2}\right)^{\delta}$. Donc on a la majoration de la hauteur de $h(\sigma(\mathscr{X}))$ dans l'énoncé. 


\subsection{Description numérique des fibres non réduites}

Soit $\overline{\mathcal{E}}$ le fibré vectoriel sur $\operatorname{Spec} \mathcal{O}_{K}$ défini dans (7). Dans la suite, on désigne par $\mathbb{P}_{K}^{n}=\mathbb{P}\left(\mathcal{E}_{K}\right)$ et $\mathbb{P}_{\mathcal{O}_{K}}^{n}=\mathbb{P}(\mathcal{E})$ pour simplifier. Soit $X \hookrightarrow \mathbb{P}_{K}^{n}$ l'hypersurface définie par un polynôme homogène

$$
f\left(T_{0}, \ldots, T_{n}\right)=\sum_{\substack{\left(i_{0}, \ldots, i_{n}\right) \in \mathbb{N}^{n+1} \\ i_{0}+\cdots+i_{n}=\delta}} a_{i_{0}, \ldots, i_{n}} T_{0}^{i_{0}} \cdots T_{n}^{i_{n}} \in K\left[T_{0}, \ldots, T_{n}\right]
$$

de degré $\delta$, et $\mathscr{X}$ l'adhérence schématique de $X$ dans $\mathbb{P}_{\mathcal{O}_{K}}^{n}$. Soit $F \in \mathbb{A}_{\mathcal{O}_{K}}\left[T_{0}, \ldots, T_{n}\right]$ un polynôme adéliquement primitif associé à $f$ comme construit dans (18) de $\S 4$.2. Pour chaque idéal maximal $\mathfrak{p} \in \operatorname{Spm} \mathcal{O}_{K}$, la réduction de $\mathscr{X}$ modulo $\mathfrak{p}$ se factorise par la localisation de $\mathcal{O}_{K}$ vers $\mathcal{O}_{K, \mathfrak{p}}$. En effet, on a le diagramme cartésien

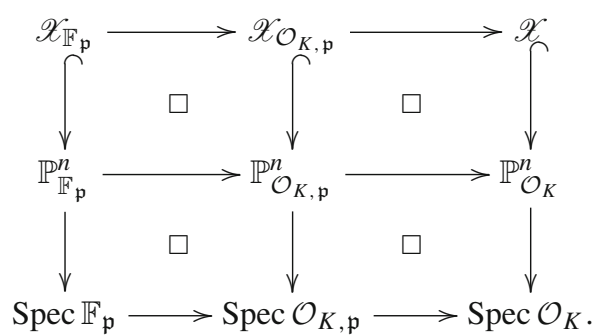

Par définition, $\mathscr{X}_{\mathcal{O}_{K, \mathfrak{p}}} \hookrightarrow \mathbb{P}_{\mathcal{O}_{K, \mathfrak{p}}}^{n}$ est défini par la p-partie $F^{(\mathfrak{p})}\left(T_{0}, \ldots, T_{n}\right)$ (voir la Définition 3.9) de $F\left(T_{0}, \ldots, T_{n}\right)$ au-dessus, qui est primitif sur $\mathcal{O}_{K, \mathfrak{p}}$.

Avec les résultats ci-dessus, on va démontrer le résultat suivant.

Theorem 5.5 Soient $X \hookrightarrow \mathbb{P}_{K}^{n}$ une hypersurface réduite, et $\mathscr{X}$ l'adhérence schématique de $X$ dans $\mathbb{P}_{\mathcal{O}_{K}}^{n}$. Soit $N(\mathfrak{p})=\#\left(\mathcal{O}_{K} / \mathfrak{p}\right)$, où $\mathfrak{p} \in \operatorname{Spm} \mathcal{O}_{K}$. Avec les notations au-dessus, on a l'inégalité

$$
\frac{1}{[K: \mathbb{Q}]} \sum_{\mathfrak{p} \in \mathcal{Q}(\mathscr{X})} \log N(\mathfrak{p}) \leq(2 \delta-1) h(X)+C_{1}(n, \delta),
$$

où la constante

$$
\begin{aligned}
C_{1}(n, \delta)= & (2 \delta-1) \delta \log \left(\frac{\delta+1}{2}\right)+\log \left(2 \delta^{\delta}-\delta^{\delta-1}\right)+(3 \delta-2) \log \left(\begin{array}{c}
n+\delta \\
n
\end{array}\right) \\
& +(2 \delta-1) \log \left(\begin{array}{c}
\delta \\
{\left[\frac{\delta+1}{2}\right]}
\end{array}\right),
\end{aligned}
$$

la notation $\mathcal{Q}(\mathscr{X})$ est dans (22), et $h(X)$ est définie dans la Définition 3.2.

Proof Si $\delta=1$, alors $X$ est un hyperplan dans $\mathbb{P}_{K}^{n}$. Dans ce cas-là, on a

$$
\frac{1}{[K: \mathbb{Q}]} \sum_{\mathfrak{p} \in \mathcal{Q}(\mathscr{X})} \log N(\mathfrak{p})=0
$$

par la définition directement, qui satisfait l'assertion.

Dans la suite on suppose $\delta \geq 2$. On choisit un élément $\sigma \in \operatorname{Aut}_{\mathcal{O}_{K}}\left(\mathbb{P}_{\mathcal{O}_{K}}^{n}\right)=\operatorname{PGL}_{n}\left(\mathcal{O}_{K}\right)$, qui envoie la coordonnée $T_{i}$ dans $T_{i}+a_{i} T_{n}$ pour les $i=0, \ldots, n-1$ où $a_{i} \in \mathbb{Z}$, et envoie 
$T_{n}$ dans $T_{n}$. Par le Lemme 5.3, on a $\mathcal{Q}(\mathscr{X})=\mathcal{Q}(\sigma(\mathscr{X}))$. De plus, d'après le Lemme 5.4 et le calcul dans (23), il existe un $\sigma \in \operatorname{Aut}_{\mathcal{O}_{K}}\left(\mathbb{P}_{\mathcal{O}_{K}}^{n}\right)$, tel que

$$
h(\sigma(\mathscr{X})) \leq h(X)+\log \left(\left(\begin{array}{c}
n+\delta \\
\delta
\end{array}\right)\left(\begin{array}{c}
\delta \\
{\left[\frac{\delta+1}{2}\right]}
\end{array}\right)\left(\frac{\delta+1}{2}\right)^{\delta}\right)
$$

et la fibre générique de $\sigma(\mathscr{X})$ soit définie par un polynôme homogène dont le coefficient du terme $T_{n}^{\delta}$ ne soit pas zéro.

On suppose la fibre générique de $\sigma(\mathscr{X}) \hookrightarrow \mathbb{P}_{\mathcal{O}_{K}}^{n}$ est définie par le polynôme homogène $f\left(T_{0}, \ldots, T_{n}\right)$ à coefficients dans $K$, et on écrit

$$
f\left(T_{0}, \ldots, T_{n}\right)=t_{\delta} T_{n}^{\delta}+t_{\delta-1}\left(T_{0}, \ldots, T_{n-1}\right) T_{n}^{\delta-1}+\cdots+t_{0}\left(T_{0}, \ldots, T_{n-1}\right)
$$

où $t_{\delta} \neq 0$. D'après la forme (24), on écrit le polynôme adéliquement primitif $F\left(T_{0}, \ldots, T_{n}\right) \in$ $\mathbb{A}_{\mathcal{O}_{K}}\left[T_{0}, \ldots, T_{0}\right]$ de $f$ obtenu dans $\S 4.2$ sous la forme de

$$
F\left(T_{0}, \ldots, T_{n}\right)=t_{\delta}^{\prime} T_{n}^{\delta}+t_{\delta-1}^{\prime}\left(T_{0}, \ldots, T_{n-1}\right) T_{n}^{\delta-1}+\cdots+t_{0}^{\prime}\left(T_{0}, \ldots, T_{n-1}\right),
$$

où tous les $t_{i}^{\prime}$ sont obtenus par la multiplication de $t_{i}$ par $c \in \mathbb{A}_{K}$ dans (24) pour les $i=$ $0, \ldots, \delta$.

On considère $F\left(T_{0}, \ldots, T_{n}\right)$ comme un polynôme de variable $T_{n}$ sur l'anneau $\mathbb{A}_{\mathcal{O}_{K}}\left[T_{0}, \ldots, T_{n-1}\right]$ de degré $\delta$. Comme $X$ est réduit, alors $F\left(T_{0}, \ldots, T_{n}\right)$ n'a pas de facteur carré d'après la Remarque 5.2. Donc pour tout $\mathfrak{p} \in \operatorname{Spm}_{\mathcal{O}_{K}}$, on a $\operatorname{Res}_{\delta}^{\prime}\left(F, \frac{\partial F}{\partial T_{n}}\right)^{(\mathfrak{p})} \neq 0$ (voir (17) pour la définition de ce résultant). Donc si $F^{(\mathfrak{p})}\left(T_{0}, \ldots, T_{n}\right)$ modulo $\mathfrak{p}\left[T_{0}, \ldots, T_{n}\right]$ admet un facteur carré à la variable $T_{n}$, le polynôme $\operatorname{Res}_{\delta}^{\prime}\left(F, \frac{\partial F}{\partial T_{n}}\right)^{(\mathfrak{p})}$ modulo $\mathfrak{p}\left[T_{0}, \ldots, T_{n-1}\right]$ s'annule.

Si $F\left(T_{0}, \ldots, T_{n}\right)$ modulo $\mathfrak{p}\left[T_{0}, \ldots, T_{n}\right]$ a un facteur carré sans variable $T_{n}$, on a $\mathfrak{p} \in$ $\mathcal{P}\left(t_{\delta}^{\prime}\right)=\left\{\mathfrak{p} \in \operatorname{Spm} \mathcal{O}_{K} \mid t_{\delta}^{\prime(\mathfrak{p})} \bmod \mathfrak{p}=0\right\}$. Donc on a

$$
\mathcal{Q}(\sigma(\mathscr{X})) \subseteq \mathcal{P}\left(t_{\delta}^{\prime}\right) \cup \mathcal{P}\left(\operatorname{Res}_{\delta}^{\prime}\left(F, \frac{\partial F}{\partial T_{n}}\right)\right),
$$

voir (20) pour la définition de $\mathcal{P}\left(\operatorname{Res}_{\delta}^{\prime}\left(F, \frac{\partial F}{\partial T_{n}}\right)\right)$.

Pour l'estimation de $\mathcal{P}\left(t_{\delta}^{\prime}\right)$, on a

$$
\begin{aligned}
\frac{1}{[K: \mathbb{Q}]} \sum_{\mathfrak{p} \in \mathcal{P}\left(t_{\delta}^{\prime}\right)} \log N(\mathfrak{p}) & \leq-\sum_{\mathfrak{p} \in \operatorname{Spm} \mathcal{O}_{K}} \frac{\left[K_{\mathfrak{p}}: \mathbb{Q}_{\mathfrak{p}}\right]}{[K: \mathbb{Q}]} \log \left|t_{\delta}^{\prime}\right|_{\mathfrak{p}} \\
& =-\sum_{v \in M_{K}} \frac{\left[K_{v}: \mathbb{Q}_{v}\right]}{[K: \mathbb{Q}]} \log \left|c t_{\delta}\right|_{v}+\sum_{v \in M_{K}, \infty} \frac{\left[K_{v}: \mathbb{Q}_{v}\right]}{[K: \mathbb{Q}]} \log \left|t_{\delta}^{\prime}\right|_{v} \\
& \leq h_{\infty}(F)=h(\sigma(F),
\end{aligned}
$$

où la ligne dernière ci-dessus est d'après (19) car $F$ est adéliquement primitif et $t_{\delta}^{\prime}$ est un coefficient de $F$, voir la Définition 3.11 pour la définition de $h_{\infty}(F)$. 
Par la relation ci-dessus, on a

$$
\begin{aligned}
& \frac{1}{[K: \mathbb{Q}]} \sum_{\mathfrak{p} \in \mathcal{Q}(\sigma(\mathscr{X}))} \log N(\mathfrak{p}) \\
& \leq \frac{1}{[K: \mathbb{Q}]} \sum_{\mathfrak{p} \in \mathcal{P}\left(\operatorname{Res}_{\delta}^{\prime}\left(F, \frac{\partial F}{\partial T_{n}}\right)\right)} \log N(\mathfrak{p})+\frac{1}{[K: \mathbb{Q}]} \sum_{\mathfrak{p} \in \mathcal{P}\left(t_{\delta}^{\prime}\right)} \log N(\mathfrak{p}) \\
& \leq(2 \delta-2) h(\sigma(\mathscr{X}))+\log \left(2 \delta^{\delta}-\delta^{\delta-1}\right)+(\delta-1) \log \left(\begin{array}{c}
\delta+n \\
n
\end{array}\right)+h(\sigma(\mathscr{X})) \\
& \leq(2 \delta-1) h(X)+\log \left(2 \delta^{\delta}-\delta^{\delta-1}\right)+(\delta-1) \log \left(\begin{array}{c}
\delta+n \\
n
\end{array}\right) \\
& \quad+(2 \delta-1) \log \left(\left(\begin{array}{c}
n+\delta \\
\delta
\end{array}\right)\left(\begin{array}{c}
\delta \\
{\left[\frac{\delta+1}{2}\right]}
\end{array}\right)\left(\frac{\delta+1}{2}\right)^{\delta}\right),
\end{aligned}
$$

où la deuxième inégalité ci-dessus est déduite à partir de la Proposition 4.1, et la dernière inégalité est du Lemme 5.4. Donc on a l'assertion.

\section{Contrôle des fibres non réduites d'un schéma de dimension pure}

Dans cette section ,on fixe $\overline{\mathcal{E}}$ le fibré vectoriel sur $\operatorname{Spec} \mathcal{O}_{K}$ défini dans (7), et on désigne $\mathbb{P}_{K}^{n}=\mathbb{P}\left(\mathcal{E}_{K}\right)$ et $\mathbb{P}_{\mathcal{O}_{K}}^{n}=\mathbb{P}(\mathcal{E})$ pour simplifier. Soit $X$ un sous-schéma fermé de dimension pure de $\mathbb{P}_{K}^{n}$, qui est de dimension $d$ et degré $\delta$. On désigne par $\mathscr{X}$ l'adhérence schématique de $X$ dans $\mathbb{P}_{\mathcal{O}_{K}}^{n}$. Dans cette section, on contrôlera les réductions non réduites de $\mathscr{X}$ lorsque $X$ est réduit.

D'après la Proposition 2.7, la formation du diviseur de Cayley de $\mathscr{X} \hookrightarrow \mathbb{P}_{\mathcal{O}_{K}}^{n}$ commute au changement de base de $\mathcal{O}_{K}$ vers un corps résiduel, voir $\S 2.1$ pour la formation sur un corps, et $\S 2.2$ pour la formation $\operatorname{sur} \operatorname{Spec} \mathcal{O}_{K}$. Donc pour côntroler les fibres non réduites de $\mathscr{X} \rightarrow \operatorname{Spec} \mathcal{O}_{K}$, d'après la Proposition 2.2, on a besoin de considérer les réduction du diviseur de Cayley de $\mathscr{X}$ telles que les variétés de Cayley correspondantes ne soient pas réduites.

Par l'argument ci-dessus, on a le côntrole des fibres non réduites de $\mathscr{X}$ suivant.

Theorem 6.1 Soient $X$ un sous-schéma fermé réduit de dimension pure de $\mathbb{P}_{K}^{n}$, qui est de dimension d et de degré $\delta$, et $\mathscr{X}$ l'adhérence schématique $X$ dans $\mathbb{P}_{\mathcal{O}_{K}}$. De plus, soient $N=\left(\begin{array}{l}n+1 \\ d+1\end{array}\right)-1, \mathcal{H}_{N}=1+\frac{1}{2}+\cdots+\frac{1}{N}$, et $\overline{\mathcal{O}(1)}$ muni des métriques de Fubini-Study induites par le fibré vectoriel $\overline{\mathcal{E}}$ défini à $(7)$, et $h \overline{\mathcal{O}(1)}(\mathscr{X})$ est la hauteur arakelovienne de $X$ définie dans la Définition 3.1. Alors on a

$$
\frac{1}{[K: \mathbb{Q}]} \sum_{\mathfrak{p} \in \mathcal{Q}(\mathscr{X})} \log N(\mathfrak{p}) \leq(2 \delta-1) h_{\overline{\mathcal{O}(1)}}(\mathscr{X})+C_{2}(n, d, \delta),
$$

où $\mathcal{Q}(\mathscr{X})$ est défini dans (22), et la constante

$$
\begin{aligned}
C_{2}(n, d, \delta)= & (3 \delta-2) \log \left(\begin{array}{c}
N+\delta \\
N
\end{array}\right)+(2 \delta-1) \delta \log \frac{\delta+1}{2}+(2 \delta-1) \log \left(\begin{array}{c}
\delta \\
{\left[\frac{\delta+1}{2}\right]}
\end{array}\right) \\
& +\log \left(2 \delta^{\delta}-\delta^{\delta-1}\right)+(2 \delta-1)\left(4 \delta \log (N+1)+(N+1) \delta \log 2-\frac{1}{2} \delta \mathcal{H}_{N}\right) .
\end{aligned}
$$


Proof Soit $\Psi_{\mathscr{X}}$ le diviseur de Cayley de $\mathscr{X}$ prolongé dans $\mathbb{P}_{\mathcal{O}_{K}}^{n}$. Car $\mathscr{X} \rightarrow \operatorname{Spec} \mathcal{O}_{K}$ est plat, donc $\Psi_{\mathscr{X}}$ est plat aussi sur $\operatorname{Spec} \mathcal{O}_{K}$ par la Proposition 2.5. Pour tout $\mathfrak{p} \in \operatorname{Spm} \mathcal{O}_{K}$, soit $\Psi_{\mathscr{X}, \mathbb{F}_{\mathfrak{p}}}$ la variété de Cayley de $\mathscr{X}_{\mathbb{F}_{\mathfrak{p}}} \hookrightarrow \mathbb{P}_{\mathbb{F}_{\mathfrak{p}}}^{n}$ définie dans la Définition 2.3 à partir de $\mathscr{X} \hookrightarrow \mathbb{P}_{\mathcal{O}_{K}}^{n}$ par la réduction à $\mathfrak{p}$. Alors d'après la Proposition 2.7, on a

$$
\Psi_{\mathscr{X}} \times \operatorname{Spec} \mathcal{O}_{K} \operatorname{Spec} \mathbb{F}_{\mathfrak{p}}=\left[\Psi_{\mathscr{X}, \mathbb{F}_{\mathfrak{p}}}\right]
$$

comme diviseurs sur $\mathbb{P}\left(\bigwedge^{d+1} \mathcal{E}_{\mathbb{F}_{\mathfrak{p}}}\right)$. Donc $\Psi_{\mathscr{X}} \times_{\operatorname{Spec}} \mathcal{O}_{K}$ Spec $\mathbb{F}_{\mathfrak{p}}$ détermine la même hypersurface que $\Psi_{\mathscr{X}, \mathbb{F}_{\mathfrak{p}}}$ dans $\mathbb{P}\left(\bigwedge^{d+1} \mathcal{E}_{\mathbb{F}_{\mathfrak{p}}}\right)$.

On désigne

$$
\mathcal{Q}\left(\Psi_{\mathscr{X}}\right)=\left\{\mathfrak{p} \in \operatorname{Spm} \mathcal{O}_{K} \mid \Psi_{\mathscr{X}, \mathbb{F}_{\mathfrak{p}}} \text { ne soit pas réduit }\right\} .
$$

Alors d'après la Proposition 2.7, la Proposition 2.2 et la Remarque 5.2, l'idéal maximal $\mathfrak{p} \in \mathcal{Q}\left(\Psi_{\mathscr{X}}\right)$ si et seulement si le schéma $\mathscr{X} \times_{\operatorname{Spec}} \mathcal{O}_{K} \operatorname{Spec} \mathbb{F}_{\mathfrak{p}}$ n'est pas réduit, d'où l'on a

$$
\frac{1}{[K: \mathbb{Q}]} \sum_{\mathfrak{p} \in \mathcal{Q}(\mathscr{X})} \log N(\mathfrak{p})=\frac{1}{[K: \mathbb{Q}]} \sum_{\mathfrak{p} \in \mathcal{Q}\left(\Psi_{\mathscr{X}}\right)} \log N(\mathfrak{p}),
$$

voir les notations ci-dessus dans (22).

D'après la Proposition 2.7 aussi, le cycle $\Psi_{\mathscr{X}} \times_{\operatorname{Spec}} \mathcal{O}_{K}$ Spec $K$ détermine une hypersurface de $\mathbb{P}\left(\bigwedge^{d+1} \mathcal{E}_{K}\right)$ de degré $\delta$. On prend un élément $\psi_{X} \in \operatorname{Sym}_{K}^{\delta}\left(\bigwedge^{d+1} \mathcal{E}_{K}\right)$ qui définit l'hypersurface ci-dessus. Par le Théorème 5.5, on obtient

$$
\begin{aligned}
& \frac{1}{[K: \mathbb{Q}]} \sum_{\mathfrak{p} \in \mathcal{Q}\left(\Psi_{\mathscr{X}}\right)} N(\mathfrak{p}) \\
& \leq(2 \delta-1) h\left(\psi_{X}\right)+\log \left(2 \delta^{\delta}-\delta^{\delta-1}\right)+(3 \delta-2) \log \left(\begin{array}{c}
N+\delta \\
N
\end{array}\right) \\
& \quad+(2 \delta-1) \delta \log \frac{\delta+1}{2}+(2 \delta-1) \log \left(\begin{array}{c}
\delta \\
{\left[\frac{\delta+1}{2}\right]}
\end{array}\right),
\end{aligned}
$$

où $h\left(\psi_{X}\right)$ est la hauteur classique définie dans la Définition 3.2. On compare $h\left(\psi_{X}\right)$ et $h \overline{\mathcal{O}(1)}(\mathscr{X})$ dans la Proposition 3.7 pour $\overline{\mathcal{E}}$ défini à (7), et on obtient l'assertion en l'appliquant à $(25)$ et (26).

Remarque 6.2 On considère la constante $C_{2}(n, d, \delta)$ définie dans le Théorème 6.1. On a

$$
C_{2}(n, d, \delta) \ll_{d, n} \delta^{2} \log \delta .
$$

Open Access This article is licensed under a Creative Commons Attribution 4.0 International License, which permits use, sharing, adaptation, distribution and reproduction in any medium or format, as long as you give appropriate credit to the original author(s) and the source, provide a link to the Creative Commons licence, and indicate if changes were made. The images or other third party material in this article are included in the article's Creative Commons licence, unless indicated otherwise in a credit line to the material. If material is not included in the article's Creative Commons licence and your intended use is not permitted by statutory regulation or exceeds the permitted use, you will need to obtain permission directly from the copyright holder. To view a copy of this licence, visit http://creativecommons.org/licenses/by/4.0/. 


\section{References}

1. Bost, J.-B., Gillet, H., Soulé, C.: Heights of projective varieties and positive Green forms. J. Am. Math. Soc. 7(4), 903-1027 (1994)

2. Bourbaki, N.: Espaces vectoriels topologiques. Chapitres 1 à 5, new éd., Masson, Paris (1981). Éléments de mathématique. [Elements of mathematics]

3. Cassels, J.W.S.: An introduction to the geometry of numbers, Classics in Mathematics. Springer, Berlin (1997). Corrected reprint of the 1971 edition

4. Chen, H.: Explicit uniform estimation of rational points I. Estimation of heights. Journal für die Reine und Angewandte Mathematik 668, 59-88 (2012)

5. Eisenbud, D.: Commutative algebra, Graduate Texts in Mathematics, vol. 150. Springer-, New York (1995). With a view toward algebraic geometry

6. Erné, R.: Reducibility mod $p$ of hypersurfaces in projective spaces—an application of arithmetic Bézout. J. Number Theory 84(2), 305-316 (2000)

7. Erné, R.: Reducibility mod $p$ of integral closed subschemes in projective spaces-an application of arithmetic Bézout. Math. Res. Lett. 7(4), 405-410 (2000)

8. Faltings, G.: Diophantine approximation on abelian varieties. Ann. Math. Second Ser. 133(3), 549-576 (1991)

9. Fukshansky, L.: Integral points of small height outside of a hypersurface. Monatsh. Math. 147(1), 25-41 (2006)

10. Fulton, W.: Intersection theory, 2nd édn. Ergebnisse der Mathematik und ihrer Grenzgebiete. 3. Folge. A Series of Modern Surveys in Mathematics [Results in Mathematics and Related Areas. 3rd Series. A Series of Modern Surveys in Mathematics], vol. 2. Springer, Berlin (1998)

11. Gel'fand, I.M., Kapranov, M.M., Zelevinsky, A.V.: Discriminants, resultants, and multidimensional determinants, Mathematics: Theory and Applications. Birkhäuser Boston Inc., Boston (1994)

12. Gillet, H., Soulé, C.: Arithmetic intersection theory, Institut des Hautes Études Scientifiques. Publications Mathématiques (1990), no. 72, pp. 93-174 (1991)

13. Grothendieck, A., Dieudonné, J.: Éléments de géométrie algébrique. IV. Étude locale des schémas et des morphismes de schémas. III, Institut des Hautes Études Scientifiques. Publications Mathématiques, no. 28 , p. 255 (1966)

14. Grothendieck, A., Dieudonné, J.: Éléments de géométrie algébrique, Die Grundlehren der mathematischen Wissenschaften in Einzeldarstellungen, vol. 166. Springer, Berlin (1971)

15. Hartshorne, R.: Algebraic Geometry, Graduate Texts in Mathematics, No. 52. Springer, New York (1977)

16. Hindry, M., Silverman, J.H.: Diophantine Geometry: An Introduction, Graduate Texts in Mathematics, vol. 201. Springer, New York (2000)

17. Liu, Q.: Algebraic geometry and arithmetic curves, Oxford Graduate Texts in Mathematics, vol. 6. Oxford University Press, Oxford (2002). Translated from the French by Reinie Erné, Oxford Science Publications

18. Neukirch, J.: Algebraic number theory, Grundlehren der Mathematischen Wissenschaften [Fundamental Principles of Mathematical Sciences], vol. 322. Springer, Berlin (1999) [Translated from the 1992 German original and with a note by Norbert Schappacher. With a foreword by G. Harder]

19. Philippon, P.: Critères pour l'indépendance algébrique, Institut des Hautes Études Scientifiques. Publications Mathématiques 64, 5-52 (1986)

20. Philippon, P.: Sur des hauteurs alternatives. I. Math. Ann. 289(2), 255-283 (1991)

21. Serre, J.-P.: Algèbre locale. Multiplicités, Cours au Collège de France, 1957-1958, rédigé par Pierre Gabriel. Seconde édition, Lecture Notes in Mathematics, vol. 11, p. 1965. Springer, Berlin (1965)

22. Soulé, C.: Lectures on Arakelov geometry, Cambridge Studies in Advanced Mathematics, vol. 33. Cambridge University Press, Cambridge (1992). With the collaboration of D. Abramovich, J.-F. Burnol and J. Kramer

Publisher's Note Springer Nature remains neutral with regard to jurisdictional claims in published maps and institutional affiliations. 